\title{
Entropy Production by Block Variable Summation and Central Limit Theorems
}

\author{
E. A. Carlen ${ }^{\star}$ and A. Soffer ${ }^{\star} \star$ \\ Department of Mathematics, Princeton University, Princeton, NJ 08544, USA \\ Received April 27, 1990; in revised form February 22, 1991
}

\begin{abstract}
We prove a strict lower bound on the entropy produced when independent random variables are summed and rescaled. Using this, we develop an approach to central limit theorems from a dynamical point of view in which the entropy is a Lyapunov functional governing approach to the Gaussian limit. This dynamical approach naturally extends to cover dependent variables, and leads to new results in pure probability theory as well as in statistical mechanics. It also provides a unified framework within which many previous results are easily derived.
\end{abstract}

\section{Introduction}

We develop an approach to central limit theorems for dependent as well as independent random variables based on ideas from dynamical systems and information theory, and we obtain new results in statistical mechanics and probability theory using it. The approach is motivated in part by Jona-Lasinio's study of the relation between renormalization group methods in statistical mechanics and the classical limit theorems of probability [JoL], and in part by Linnik's information-theoretic proof of the central limit theorem [Lin59]. In renormalization group analysis, one considers block summation of random variables as an iterative dynamics on the state space of a dynamical system whose states are certain arrays of random variables [Gaw]. What we show here is that entropy often serves as a good Lyapunov functional for the convergence to a Gaussian fixed point under this dynamics. This results in a clean implementation of Linnik's ideas in a broad setting with significant additional consequences.

Our results are based on a detailed study of entropy production in the process of summing independent random variables; or equivalently, the entropy produc-

\footnotetext{
$\star$ Partially supported by an NSF postdoctoral fellowship

$\star \star$ Partially supported by NSF grant DMS-8905772; A. P. Sloan Fellow in Mathematics
} 
tion of convolution. We then control entropy production in the dependent case by using strict lower bounds from our study of the independent case to absorb the effects of dependence.

We first describe our results and methods, postponing to the end of this introduction most discussion of the relation between our work here and work in the existing literature. We mention now however that the literature is vast, and that we draw on it in many places.

The paper is written in three sections. The first contains our results on entropy production in the independent case. Careful definitions are to be found there, but recall that the entropy $S(X)$ of a random variable $X$ with density $\varrho$ is given by $S(X)$ $=S(\varrho)=-\int_{\mathbf{R}} \varrho \ln \varrho(x) d x$. This is always well defined when $X$ has finite variance provided we allow $-\infty$ as a possible value. For random variables $X$ of unit variance it is well known that $S(X) \leqq S(G)$, where $G$ is a unit Gaussian random variable, and there is equality only when $X$ itself is a unit Gaussian random variable.

This upper bound on the entropy is especially interesting in conjunction with the following entropy production inequality conjectured by Shannon [ShWe] and proved by Stam [Sta]: Suppose $X_{1}$ and $X_{2}$ are independent random variables with zero mean and unit variance such that $S\left(X_{1}\right), S\left(X_{2}\right)>-\infty$. Then

$$
S\left(\lambda X_{1}+\left(1-\lambda^{2}\right)^{1 / 2} X_{2}\right)-\lambda^{2} S\left(X_{1}\right)-\left(1-\lambda^{2}\right) S\left(X_{2}\right) \geqq 0 \quad \forall 0<\lambda<1
$$

and there is equality exactly when both $X_{1}$ and $X_{2}$ are unit Gaussian random variables.

Note that the random variable on the left side of this inequality again has zero mean and unit variance.

The above statement about cases of equality in $(0.1)$ provides a characterization of unit Gaussian distributions. Our main technical tool is a stability result for the Shannon-Stam inequality; we show that if the entropy difference in (0.1) is close to zero, then both $X_{1}$ and $X_{2}$ are close to being unit Gaussian random variables in the relative entropy sense: The relative entropy $D(\varrho)$ of a density $\varrho$ on $\mathbf{R}^{n}$ is defined by

$$
D(\varrho)=\int\left[\frac{\varrho}{\mathbf{R}^{n}}\right] \ln \left[\frac{\varrho}{g}\right] g(x) d^{n} x,
$$

where $g(x)$ always denotes $(2 \pi)^{-n / 2} e^{-x^{2} / 2}$. Since $\|\varrho-g\|_{L^{1}\left(\mathbf{R}^{n}, d^{n} x\right)}^{2} \leqq 2 D(\varrho)$ [Kul, Csi], the relative entropy provides a strong measure of the distance from $\varrho$ to $g$.

To state and discuss our stability result more precisely, we first recall that for an $\mathbf{R}^{n}$ valued random variable $X$ with density $\varrho$, the Fisher information of $X, I(X)$, is given by

$$
I(X)=I(\varrho)=4 \int_{\mathbf{R}^{n}}|\nabla \sqrt{\varrho}(x)|^{2} d^{n} x .
$$

The main result of the first section, contained in Theorem 1.2, is the following: Suppose $X_{1}$ and $X_{2}$ are independent centered random variables with unit variance such that

$$
I\left(X_{1}\right), I\left(X_{2}\right) \leqq I_{0}<\infty .
$$

Suppose also that there is a function $\psi$ decreasing to zero at infinity so that for all $R \geqq 0$,

$$
E 1_{\left|X_{j}\right| \geqq R}\left|X_{j}\right|^{2} \leqq \psi(R),
$$


for $j=1,2$. Suppose also that the $\lambda$ in (0.1) satisfies $\lambda \geqq a$ for some $a>0$ and

$$
D\left(X_{1}\right) \geqq \varepsilon>0 \text {. }
$$

Then there is a $\delta_{\varepsilon}>0$ depending only on $I_{0}, \psi, a$ and $\varepsilon$ so that the entropy difference in (0.1) is at least $\delta_{\varepsilon}$.

To obtain this result, we first prove such a lower bound for random variables which are regularized by adding independent Gaussian variables to them and then renormalizing. Specifically, let $G_{1}$ and $G_{2}$ be centered unit Gaussian random variables independent of one another as well as $X_{1}$ and $X_{2}$. Fix $t>0$ and define reguarlized random variables $\widetilde{X}_{1}$ and $\widetilde{X}_{2}$ by

$$
\tilde{X}_{j}=e^{-t} X_{j}+\left(1-e^{-2 t}\right)^{1 / 2} G_{j} \quad j=1,2 .
$$

We are able to utilize the stability result for regularized random variables, even though the bounds it provides would appear to depend on the regularization, on account of the following monotonicity result contained in Theorem 1.1; For any $0<\lambda<1$,

$$
\begin{aligned}
& S\left(\lambda X_{1}+\left(1-\lambda^{2}\right)^{1 / 2} X_{2}\right)-\lambda^{2} S\left(X_{1}\right)-\left(1-\lambda^{2}\right) S\left(X_{2}\right) \\
& \quad \geqq S\left(\lambda \tilde{X}_{1}+\left(1-\lambda^{2}\right)^{1 / 2} \tilde{X}_{2}\right)-\lambda^{2} S\left(\tilde{X}_{1}\right)-\left(1-\lambda^{2}\right) S\left(\tilde{X}_{2}\right)
\end{aligned}
$$

whenever the top line is well defined; moreover, there is equality just when $X_{1}$ and $X_{2}$ are unit Gaussian random variables. (By increasing $t$ in (0.4) to infinity, it is easy to deduce from (0.5) the strict form of Shannon's inequality.)

The inequality $(0.5)$ is what allows us to transfer lower bounds on the entropy production for regularized random variables back to the original random variables.

The point of the Gaussian regularization is not so much the smoothing of the densities it accomplishes - $(0.2)$ already assures us more smoothness than we need, and can be relaxed - but the fact that the densities $\varrho$ of the regularized random variables satisfy

$$
|\ln \varrho(x)| \leqq A\left(1+x^{2}\right),
$$

where the constant $A$ depends only on the variable $t$ in (0.4). This enables us to show that the closure in $L^{1}\left(\mathbf{R}^{n},\left(1+x^{2}\right) d^{n} x\right)$ of the regularized densities of centered unit variance random variables satisfying $(0.3)$ is a compact set on which the entropy is a continuous function. Moreover, the entropy difference in $(0.1)$ considered as a function of a pair of densities is continuous on the product of this space with itself. From here, it is easy to establish Theorem 1.2 once one has $(0.5)$.

To prove $(0.5)$ we first prove a variant of Shannon's inequality for the Fisher information, and then derive our desired entropy inequalities form these information inequalities using a relation between entropy and information arising through the action of the adjoint Ornstein-Uhlenbeck semigroup on probability densities. This is explained and motivated at the beginning of the first section. Here we note only that the reasoning behind our proofs of these inequalities consists almost exclusively of convexity and symmetry arguments. It is useful to introduce the Fisher information because, while it is closely related to the entropy, it has better convexity properties in a sense that we will make clear.

In the second section of the paper, we prove several new central limit theorems. Because of our intended applications, and consistently with our renormalization group motivation, we consider rectangular arrays of $\mathbf{R}^{n}$ valued random variables $\left\{X_{n, j} \mid n, j \in \mathbf{N}\right\}$. Let $\mathscr{X}_{n}$ denote the discrete stochastic process $\left\{X_{n, j} \mid j \in \mathbf{N}\right\}$. Block 
summation generates the rectangular array out of $\mathscr{X}_{1}$ by iteration of the map taking $\mathscr{X}_{n}$ to $\mathscr{X}_{n+1}$ which is given by

$$
X_{n+1, j}=X_{n, 2 j-1}+X_{n, 2 j} \text {. }
$$

Let $\mathscr{Z}_{n}$ be the stochastic process parameterized by $\mathbf{N}$ in which the $j^{\text {th }}$ random variable is $X_{n, j}$ after centering and normalization. Finally, let $\mathscr{G}$ denote the stochastic process $\left\{G_{j} \mid j \in \mathbf{N}\right\}$ consisting of independent centered $n$-dimensional Gaussian variables with unit variance. We will prove theorems asserting that

$$
\lim _{n \rightarrow \infty} \mathscr{Z}_{n}=\mathscr{G}
$$

in various topologies. We remark right away that our applications will not be restricted to one dimensional statistical mechanical systems: the parameter $j$ above can run over some ordering - possibly changing with $n$ - of the points in a higher dimensional lattice.

To prove a central limit theorem for independent random variables, we need mainly to be able to apply Theorem 1.2 uniformly in all block summations. It already follows from the results of the first section that if $I\left(Z_{1, j}\right) \leqq I_{0}$ for all $j$, then $I\left(Z_{n, j}\right) \leqq I_{0}$ for all $n$ and $j$. We must also show such a result for the uniform concentration condition (0.3). This is done in Theorem 2.1. It gives conditions under which uniform concentration is propagated by the block summation dynamics, so to speak; and it amounts to an effective renormalization group approach to verifying a Lindeberg condition. The result is applicable even for some fairly strongly dependent variables as well as independent variables. In the case of independent variables, it is then easy to give conditions on $\mathscr{Z}_{1}$ so that with $s_{n}=\inf \left\{S\left(Z_{n, j}\right) \mid j \in \mathbf{N}\right\}$,

$$
s_{n+1} \geqq \min \left\{(S(g)-\varepsilon),\left(s_{n}+\delta_{\varepsilon}\right)\right\},
$$

and this leads, under conditions ensuring that $s_{1}>-\infty$, to $\lim _{n \rightarrow \infty} s_{n}=S(g)$. Hence (0.6) holds with all the marginal distributions converging in the relative entropy sense.

This may seem like a roundabout way of exploiting the strict entropy increase provided the Shannon-Stam inequality. After all, (0.1) implies

$$
S\left(2^{-n / 2} \sum_{j=1}^{2^{n}} X_{j}\right)
$$

is strictly monotone increasing in $n$ for an i.i.d. sequence of centered random variables with unit variance and finite entropy less than $S(g)$. But then even knowing the usual central limit theorem, one cannot conclude from this that it increases all the way to $S(g)$ because the entropy is only a weakly upper semicontinuous function on $L^{1}\left(\mathbf{R}^{n}, d^{n} x\right)$.

The remaining results concern dependent random variables, but with convergence in the usual distributional sense. Think of each $X_{j, n}$ as representing a block sum of random variables from some fixed underlying spin system. Let the $Z_{n, j}$ be defined as above. When the blocks become large in such a setting, the block variables $X_{n, j}$ and $X_{n, k}$ are often only weakly dependent. Consider the entropy difference

$$
S\left(X_{n, 2 j-1}+X_{n, 2 j} / \sigma\left(X_{n, 2 j-1}+X_{n, 2 j}\right)\right)-\left(S\left(Z_{n, 2 j-1}\right)+S\left(Z_{n, 2 j}\right)\right) / 2
$$


to which Theorem 1.2 does not apply on account of the dependence, however weak. But let $Y_{n, 2 j-1}$ denote a copy of $Z_{n, 2 j-1}$ which is independent of everything else. Then since clearly $S\left(Z_{n, 2 j-1}\right)=S\left(Y_{n, 2 j-1}\right)$, we can bound (0.7) from below by

$$
\begin{aligned}
& S\left(\left(Y_{n, 2 j-1}+Z_{n, 2 j}\right) / \sqrt{2}\right)-\left(S\left(Y_{n, 2 j-1}\right)+S\left(Z_{n, 2 j}\right)\right) / 2 \\
& \quad-\left|S\left(\left(Y_{n, 2 j-1}+Z_{n, 2 j}\right) / \sqrt{2}\right)-S\left(\left(X_{n, 2 j-1}+X_{n, 2 j}\right) / \sigma\left(X_{n, 2 j-1}+X_{n, 2 j}\right)\right)\right| .
\end{aligned}
$$

Suppose, for convenience, that for large $n$ all the random variables in $\mathscr{X}_{n}$ have nearly the same variance so that $1 / \sqrt{2}$ would be the correct value of $\lambda$ for application of Theorem 1.2 in the independent case. Then we can show that if the random variables in $\mathscr{X}_{n}$ are only weakly dependent for large $n$, the second term above tends to zero with increasing $n$. The notion of "weak dependence" here is fairly mild. Then using Theorem 1.2 we can show that the entropy increase given by the first term is bounded below as long as $Z_{n, 2 j-1}$ and $Z_{n, 2 j}$ are not both close to being unit Gaussians. Thus for large $n$, the entropy increase of adding up independent blocks dominates the entropy loss produced by then coupling them. Now one obtains central limit theorems essentially as in the independent case.

However, since we want central limit theorems for discrete random variables, such as Ising spins, which do not posses a density, we first make a Gaussian regularization of our random variables, and then employ the strategy just described. But it is easy to see that in case the regularized random variables converge to Gaussians in the relative entropy sense for every regularization, then the original variables converge to these Gaussians in distribution. By giving up some strength of the sense of convergence, we can extend our treatment to random variables for which the entropy is not finite. In this way we obtain Theorems 2.3, 2.4 and 2.5. The last two results do not refer to entropy in either their hypotheses or conclusions. Thus although we prove them here using entropy inequalities, they could be provable by other methods. However, their particular formulations, which turn out to be quite useful, were suggested by our proof of Theorem 2.2. Moreover, we learn in our approach not only that the Gaussian limit holds, but that entropy works as a Lyapunov functional governing convergence to this limit in statistical mechanical settings.

In the third section we apply our central limit theorem for sums of dependent random variables to prove new central limit theorems for extensive variables in a number of lattice spin systems. There is a large literature on this subject. We refer in particular to [Dec, GaJl, GaMl, Ne80 and Ne83]; but see the bibliographies of these papers as well.

Our method is essentially non-perturbative so that we only need to control a few correlation functions. In many cases finite susceptibility, i.e. summability of the truncated two point function, suffices, as in Newman's [Ne80] result for translation invariant FKG systems. However we do not always need the FKG inequalities. We can treat several multicomponent systems for which, due to the lack of an ordering on the spin space, there is no FKG inequality. One aspect setting our work apart from other non-perturbative approaches is that we can often avoid any translation invariance requirement - even stochastic - so that our method should be useful for proving central limit theorems for certain random field systems. Also, our method applies in essentially the same way to other extensive variables such as energy, for example, and joint distributions of extensive variables can be handled as well. 
We shall only demonstrate the utility of our approach by applying it in a number of cases where existing results cannot be used, but shall not attempt an exhaustive catalog of possible applications. Many of the most interesting applications may be to percolation and related phenomena; that is, to problems other than those arising in spin systems as such. Indeed, we consider the fact that entropy is often a good Lyaponov functional for block summation to be our main result, and not so much the particular limit theorems we prove here for specific classes of spin systems.

We now briefly discuss some related work. The Shannon-Stam inequality occurs most frequently in the electrical engineering literature where it is simply called Shannon's entropy power inequality, and - as the name suggests - is usually written in another form. For the equivalence, see Lieb [Li75] who rediscovered it and proposed that it might be useful in statistical mechanics.

While no stability result for Shannon's inequality appears to have been known, stability results for other characterizations of the Gauss law, such as Cramér's [Cra], have been extensively studied. See the papers of Sapagov [Sap] and Shimizu [Shi82] in particular.

As noted above, (0.1) was first proved by Stam [Sta] who used the Fisher information and derived entropy inequalities from information inequalities as we do here. However, he used a quite different argument based on the relation between entropy and Fisher information arising through the action of the heat semigroup on probability densities. Our use of the Ornstein-Uhlenbeck semigroup has the distinct advantage of leading to the monotonicity inequality $(0.5)$ which did not occur in previous work.

Lieb used an entirely different method to prove the Shannon-Stam inequality. His is the only proof not involving Fisher information, but it does not settle the cases of equality.

The relation between entropy and information arising from the OrnsteinUhlenbeck semigroup is the integral identity of Lemma 1.2. It was first noted by Bakry and Emery [BaEm], and was rediscovered in an equivalent form by Barron [Bar] in work we discuss below.

In proving Theorem 2.1, we have drawn on some ideas of Brown who proved a related result for i.i.d. variables [Bro].

The use of entropy and information in the proof of central limit theorems goes back to the fundamental papers of Linnik [Lin59, Lin60]. His results differ from ours in many ways, two of which we point out: First, he only considers independent random variables, and he only obtains convergence in the usual distributional sense, albeit under the general Lindeberg condition. He does not obtain the much stronger relative entropy convergence except, as an intermediate step, for Gaussian regularizations of bounded random variables. Second, his estimates only yield entropy increase when the variance of one random variable is sufficiently small compared to that of the other. This makes his method unsuitable for block summation where the variances are comparable.

Barron [Bar] was the first to prove a central limit theorem with convergence in the relative entropy sense. He treated the case of independent identically distributed random variables building on previous work of Brown [Bro]. Though our approach is different, we have drawn on his ideas in several of our lemmas. Actually, part of Barron's and Brown's work overlaps with the earlier work of Shimizu [Shi74] who used Fisher information to prove the central limit theorem in the i.i.d. case. 
Finally, the relation between entropy and Fisher information has been used in a statistical mechanical context by McKean [McK] who used it to control the approach to equilibrium in a caricature of the Boltzman equation. For a recent related entropy production inequality, see Desvilettes [Des]. There is a considerable literature in which entropy is used to study the approach to equilibrium in such continuous time dynamical settings; see in particular [Hol] and [GPV]. The fact that our dynamics is intrinsically discrete, however, requires some differences in the approach.

For further discussion of information theoretic inequalities and their uses, see the paper of Dembo [Dem] and the book of Deuschel and Stroock [DeSt].

We would like to add that we have drawn useful inspiration from Elliott Lieb's work on extremals for Gaussian integral operators [Li89]. For an approach to proving the sharp Sobolev and related inequalities using a discrete dynamics, see [CaLo]. Also, monotonicity bounds depending on localization and smoothness are the main tool in modern scattering theory [SiSo1,2]. This approach was developed in scattering theory over a long time with Enss [Ens] and Mourre [Mou] making important contributions. In particular the use of strict monotonicity with respect to the free evolution to absorb the effects of interaction is due to Mourre.

\section{Entropy Production by Convolution}

This section of the paper contains our lower bounds on the entropy produced when independent random variables are summed. Since we draw on a wide variety of facts concerning entropy, Fisher information, the Ornstein-Uhlenbeck semigroup and its Lebesgue adjoint, we begin by providing some background.

Let $X$ be an $\mathbf{R}^{n}$ valued random variable on some probability space. Let $\mu$ denote the law of $X$. We say that $X$ has the density $\varrho$ in case $d \mu(x)=\varrho(x) d x$. Functions arising this way are called densities. We denote the mean of $X$ by $m(X)$ and the variance matrix of $X$ by $[\sigma(X)]$. Of course $[\sigma(X)]^{2}=E(X-m(X))(X-m(X))^{T}$. We say that $X$ has unit variance in case $[\sigma(X)]=I_{n \times n}$, the $n \times n$ identity matrix. We denote the trace of $[\sigma(X)]^{2}$ by $\sigma^{2}(X)$. If $F$ is any functional of $X$ depending only on $\mu$ or $\varrho$, we denote its value by $F(X), F(\mu)$ or $F(\varrho)$ according to our convenience. Terminology will be adapted in the same way.

Throughout this paper $g_{t}$ denotes the centered Gaussian density with variance $t$ :

$$
g_{t}(x)=(2 \pi t)^{-n / 2} e^{-x^{2} / 2 t} .
$$

We shall simply write $g$ in place of $g_{1}$.

The relative entropy of $\varrho$ with respect to $g, D(\varrho)$, is defined by

$$
D(\varrho)=\int\left[\frac{\varrho(x)}{g(x)}\right] \ln \left[\frac{\varrho(x)}{g(x)}\right] g(x) d^{n} x .
$$

By Jensen's inequality, $D(\varrho) \geqq 0$ with equality just when $\varrho=g$.

When $\varrho|\ln \varrho|$ is integrable,

$$
-\int_{\mathbf{R}^{n}} \varrho(x) \ln \varrho(x) d^{n} x=\frac{1}{2}\left(n \ln (2 \pi)+\int_{\mathbf{R}^{n}} x^{2} \varrho(x) d^{n} x\right)-D(\varrho) .
$$

For densities $\varrho$ of finite variance, we define the entropy of $\varrho, S(\varrho)$, by

$$
S(\varrho)=\frac{1}{2}\left(n \ln (2 \pi)+\int_{\mathbf{R}^{n}} x^{2} \varrho(x) d^{n} x\right)-D(\varrho)=S(g)-D(\varrho) .
$$


Clearly if $\varrho$ has zero mean and unit variance, $-\infty \leqq S(\varrho) \leqq S(g)$, and the upper bound is saturated just when $\varrho=g$. Moreover, when $\varrho$ has zero mean and unit variance, it is clear that

$$
D(\varrho)=S(g)-S(\varrho) .
$$

The following representation of $D(\varrho)$ due to Donsker and Varadhan [DoVa] plainly displays the strict convexity and weak lower semicontinuity properties of the relative entropy:

$$
D(\varrho)=\sup \left\{\int_{\mathbf{R}^{n}} \phi(x) \varrho(x) d^{n} x-\ln \left(\int_{\mathbf{R}^{n}} e^{\phi(x)} g(x) d^{n} x\right) \mid \phi \in \mathscr{C}_{b}\left(\mathbf{R}^{n}\right)\right\} .
$$

The relative entropy is one measure of the distance between $\varrho$ and $g$; another measure of this distance which we shall use is given by the relative Fisher information.

For a centered density $\varrho$ with $\sigma^{2}(\varrho)=n$ and $\sqrt{\varrho} \in H^{1}\left(\mathbf{R}^{n}\right)$, the Hilbert space of functions with square integrable distributional derivative, we define the Fisher information $I(\varrho)$ by

$$
I(\varrho)=4 \int_{\mathbf{R}^{n}}|\nabla \sqrt{\varrho(x)}|^{2} d^{n} x
$$

and we define the relative Fisher information of $\varrho, J(\varrho)$, by

$$
J(\varrho)=4 \int_{\mathbf{R}^{n}}\left|\left(\nabla+\frac{x}{2}\right) \sqrt{\varrho(x)}\right|^{2} d^{n} x .
$$

Clearly, $J(\varrho) \geqq 0$ with equality just when $\varrho=g$; note that $\left(\nabla+\frac{x}{2}\right)$ is the "lowering operator" for the harmonic oscillator hamiltonian which has $\sqrt{g(x)}$ for its ground state. Since $\sigma^{2}(\varrho)=n$ and $m(\varrho)=0, J(\varrho)=I(\varrho)-n$. Note that - at least in the presence of some mild regularity - one can write $J(\varrho)=\int_{\mathbf{R}^{n}}|\nabla \ln \varrho(x)-\nabla \ln g(x)|^{2} \varrho(x) d^{n} x$.

We shall also have use for the scaling properties of entropy and information: Since for any $a>0, a X$ has the density $(1 / a) \varrho(x / a)$,

$$
I(a X)=\frac{1}{a^{2}} I(X) .
$$

More generally, in case $A$ is any non-degenerate $n \times n$ matrix,

$$
S(A X)=S(X)+\ln |\operatorname{det}(A)| \text {. }
$$

Throughout this paper, $G_{1}, G_{2}, G_{3}, \ldots$ will denote independent random variables with density $g$ which are moreover independent of any other random variables appearing in any formulas containing them.

If $X_{1}$ and $X_{2}$ are independent random variables with densities $\varrho_{1}$ and $\varrho_{2}$ respectively, and if $0<\lambda<1$, we denote the density of $\left(\lambda X_{1}+\left(1-\lambda^{2}\right)^{1 / 2} X_{2}\right)$ by $\varrho_{1} *_{\lambda} \varrho_{2}$. Clearly $\varrho_{1} * \varrho_{\lambda} \varrho_{2}$ is the marginal of a product distribution: Define new variables $u$ and $v$ by $u=\lambda x+\left(1-\lambda^{2}\right)^{1 / 2} y$ and $v=-\left(1-\lambda^{2}\right)^{1 / 2} x+\lambda y$. Then $\varrho_{1} *_{\lambda} \varrho_{2}$ $=\int_{\mathbf{R}^{n}} \varrho_{1}(x) \varrho_{2}(y) d^{n} v$ so that

$$
\varrho_{1}{ }_{\lambda} \varrho_{2}(u)=\int_{\mathbf{R}^{n}} \varrho_{1}\left(\lambda u-\left(1-\lambda^{2}\right)^{1 / 2} v\right) \varrho_{2}\left(\left(1-\lambda^{2}\right)^{1 / 2} u+\lambda v\right) d^{n}
$$


The relation between entropy and information we use arises through the action on densities of the adjoint Ornstein-Uhlenbeck semigroup $\left\{P_{t}^{*} \mid t \geqq 0\right\}-$ to be defined below - and can be expressed roughly as

and

$$
\begin{gathered}
\frac{d}{d t} S\left(P_{t}^{*} \varrho\right)=J\left(P_{t}^{*} \varrho\right), \\
{\left[\sigma\left(P_{t}^{*} \varrho\right)\right]^{2}=I_{n \times n},}
\end{gathered}
$$

$$
\lim _{t \rightarrow \infty} P_{t}^{*} \varrho=g
$$

for centered densities $\varrho$ with unit variance. These facts were used by Bakry and Emery in their proof of Gross's logarithmic Sobolev inequality - which is equivalent to the entropy-information inequality (1.39) below.

Integration of (1.11) shall be used to translate sharp inequalities for $J(\varrho)$ into sharp inequalities for $S(\varrho)$. Since $J(\varrho)$ has a simple quadratic nature, it is easy to produce sharp inequalities governing the reduction in Fisher information when random variables are summed, while direct proofs of corresponding inequalities for entropy increase may be formidably difficult.

To fill in some necessary details, let $f$ be a bounded measurable function on $\mathbf{R}$. We define an operator $P_{t}, t>0$, by

$$
P_{t} f(x)=E f\left(e^{-t} x+\left(1-e^{-2 t}\right)^{1 / 2} G\right) .
$$

It is easy to see that $P_{t}\left(P_{s} f\right)(x)=P_{s+t} f(x)$, and that each $P_{t}$ extends to a contraction on each $L^{p}\left(\mathbf{R}^{n}, g(x) d^{n} x\right)$ for $1 \leqq p \leqq \infty$. $\left\{P_{t} \mid t \geqq 0\right\}$ is the OrnsteinUhlenbeck semigroup and is strongly continuous on each $L^{p}\left(\mathbf{R}^{n}, g(x) d^{n} x\right)$ for $1 \leqq p<\infty$, as well as on $\mathscr{C}_{0}\left(\mathbf{R}^{n}\right)$, the space of continuous functions on $\mathbf{R}^{n}$ which vanish at infinity.

The Lebesgue adjoint to this semigroup is defined as usual. For any $f \in L^{1}\left(\mathbf{R}^{n}, d^{n} x\right)$ and $t>0$, there is a unique function $P_{t}^{*} f \in L^{1}\left(\mathbf{R}^{n}, d^{n} x\right)$ so that

$$
\int_{\mathbf{R}^{n}} P_{t}^{*} f(x) \phi(x) d^{n} x=\int_{\mathbf{R}^{n}} f(x) P_{t} \phi(x) d^{n} x \quad \forall \phi \in L^{\infty}\left(\mathbf{R}^{n}\right) .
$$

Clearly $\left\{P_{t}^{*} \mid t \geqq 0\right\}$ is a contraction semigroup on $L^{1}\left(\mathbf{R}^{n}, d^{n} x\right)$ preserving positivity and integrals. Two useful formulas for $P_{t}^{*} f(x)$ are easily derived from (1.14) and (1.15):

and

$$
P_{t}^{*} f(x)=E e^{t}\left(f\left(e^{t}\left(x-\left(1-e^{-2 t}\right)^{1 / 2} G\right)\right)\right)
$$

$$
P_{t}^{*} f(x)=\int_{\mathbf{R}^{n}} f(y) g_{\left(1-e^{-2 t}\right)^{1 / 2}}\left(x-e^{-t} y\right) d^{n} y .
$$

In particular, if $X$ is a random variable with density $\varrho$,

$$
P_{t}^{*} \varrho(x)=E g_{\left(1-e^{-2 t}\right)^{1 / 2}}\left(x-e^{-t} X\right) \text {. }
$$

Note that $P_{t}^{*} \varrho$ is the density of $e^{-t} X+\left(1-e^{-2 t}\right)^{1 / 2} G$. Also, we can use (1.18) to define $P_{t}^{*} \mu$ for any Borel probability measure $\mu$ by taking for $X$ any random variable with this law. The estimates in the following lemma are simple OrnsteinUhlenbeck variants of heat semigroup estimates which can be found in [Bar] and [Bro].

Lemma 1.1. Suppose $\varrho$ is a density of the form $\varrho=P_{t}^{*} \mu$ for some $t>0$ and some Borel probability measure $\mu$ with zero mean and unit variance. Then there exist finite 
constants $A_{t}, B_{t}, C_{m, t}$ depending only on the dimension $n$ and the indicated parameters such that

and

$$
\begin{aligned}
& \varrho(x) \geqq A_{t} g_{\left(1-e^{-t}\right)^{1 / 2}}(x), \\
& |\nabla \sqrt{\varrho(x)}|^{2} \leqq B_{t} P_{t}^{*} \varrho(x),
\end{aligned}
$$

$$
\sum_{i_{1} \ldots i_{m}}\left|\left(\frac{\partial^{m}}{\partial x_{i_{1}} \ldots \partial x_{i_{m}}}\right) \varrho(x)\right| \leqq C_{m, t} P_{t}^{*} \varrho(x) \quad \forall m \geqq 0
$$

uniformly in $x \in \mathbf{R}^{n}$.

Proof. Let $X$ be a random variable with law $\mu$. Since $X$ has unit variance, $\operatorname{Pr}\{|X|$ $\leqq \sqrt{2 n}\} \geqq 1 / 2$. Also for any $t>s>0$ and any $x$ and $y$ in $\mathbf{R}$ with $y^{2} \leqq 2 n, g_{t}(x+y)$ $\geqq A_{s, t}(x)$, where $A_{s, t}$ depends only on $s, t$ and $n$. From (1.18) we then have

$$
\varrho(x) \geqq E 1_{\{|X| \leqq \sqrt{2 n\}}} g_{\left(1-e^{-2 t}\right)^{1 / 2}}\left(x-e^{-t} X\right) \geqq A_{s,\left(1-e^{-2 t}\right)^{1 / 2}} g_{s}(x)
$$

for any $0<s<\left(1-e^{-2 t}\right)^{1 / 2}$; taking $s=\left(1-e^{-t}\right)^{1 / 2}$ yields $(1.19)$.

Note that (1.20) is equivalent to $|\nabla \varrho(x)|^{2} \leqq 4 B_{t} \varrho P_{t}^{*} \varrho(x)$. From $(1.18)$ it is easy to see that $\varrho$ is continuously differentiable with

$$
\nabla \varrho(x)=\frac{-1}{\left(1-e^{-2 t}\right)^{1 / 2}} E\left(x-e^{-t} X\right) g_{\left(1-e^{-2 t}\right)^{1 / 2}}\left(x-e^{-t} X\right) .
$$

Thus by Schwarz's inequality

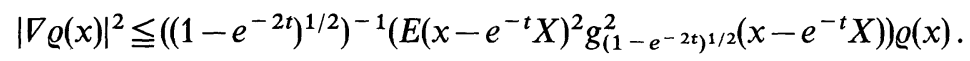

But $\left(\left(1-e^{-2 t}\right)^{1 / 2}\right)^{-1} y^{2} g_{\left(1-e^{-2 t}\right)^{1 / 2}}^{2}(y) / g_{\left(1-e^{-4 t}\right)^{1 / 2}}^{2}(y)$ is clearly bounded above by a constant depending only on $t>0$.

The final set of inequalities follow in a similar, but even simpler, fashion from the fact that for any $s>0$,

$$
\frac{\partial^{m}}{\partial x_{j}^{m}} g_{s}(x)=(\sqrt{s})^{m} H_{m}\left(x_{j} / \sqrt{s}\right) g_{s}(x),
$$

where $H_{m}$ is the $m^{\text {th }}$ Hermite polynomial.

The next result, under additional regularity assumptions, is due to Bakry and Emery. See $[\mathrm{BaEm}]$ or [DeSt]. An equivalent result in the general case can be found in [Bar], but that proof doesn't take full advantage of the available convexity, and the details are relegated to an inaccessable technical report.

Lemma 1.2. Suppose $\varrho$ is a centered density with $\sigma^{2}(\varrho)=n$. Then the map $t \mapsto S\left(P_{t}^{*} \varrho\right)$ is continuous and monotone increasing on $[0, \infty)$ with

$$
\lim _{t \rightarrow \infty} S\left(P_{t}^{*} \varrho\right)=S(g) \text {. }
$$

Furthermore, when $S(\varrho)>-\infty, t \mapsto S\left(P_{t}^{*} \varrho\right)$ is continuously differentiable on $(0, \infty)$ and

In particular,

$$
S\left(P_{t}^{*} \varrho\right)=S(\varrho)+\int_{0}^{t} J\left(P_{s}^{*} \varrho\right) d s .
$$

$$
D(\varrho)=\int_{0}^{\infty} J\left(P_{t}^{*} \varrho\right) d t .
$$


Proof. Let $\Phi_{\varrho}(\phi)$ denote $\int_{\mathbf{R}^{n}} \phi \varrho d^{n} x-\ln \left(\int_{\mathbf{R}^{n}} e^{\phi} g d^{n} x\right)$. Then by (1.15) we have

$$
\Phi_{P_{t}^{*} \varrho}(\phi)=\Phi_{\varrho}\left(P_{t} \phi\right)-\left(\ln \left(\int_{\mathbf{R}^{n}} e^{\phi_{g}} g d^{n} x\right)-\ln \left(\int_{\mathbf{R}^{n}} e^{P_{t} \phi} g d^{n} x\right)\right)
$$

for any bounded continuous function $\phi$. Notice that the right side of the above inequality is unchanged if we add a constant to $\phi$; we may therefore assume that $\phi$ is non-negative. In that case,

$$
\int_{\mathbf{R}^{n}} e^{\phi} g d^{n} x \geqq \int_{\mathbf{R}^{n}} e^{P_{t} \phi} g d^{n} x,
$$

since $P_{t}$ is a contraction on each $L^{m}\left(\mathbf{R}^{n}, g(x) d^{n} x\right)$. It then follows from (1.25) that

$$
\Phi_{P_{t}^{*}}(\phi) \leqq \Phi_{\varrho}\left(P_{t} \phi\right),
$$

and thus since $P_{t} \mathscr{C}_{b}\left(\mathbf{R}^{n}\right) \subset \mathscr{C}_{b}\left(\mathbf{R}^{n}\right)$ it follows from (1.5) that $D\left(P_{t}^{*} \varrho\right) \leqq D(\varrho)$. This shows that under $P_{t}^{*}$ the relative entropy is monotone decreasing, which by (1.4) means that the entropy itself is monotone increasing.

Similarly, to establish the continuity of $S\left(P_{t}^{*} \varrho\right)$ we first suppose that $D(\varrho)<\infty$. To establish continuity at $t=0$, it suffices to show that for any $\varepsilon>0$ there is a $t>0$ such that $D\left(P_{t}^{*} \varrho\right) \geqq D(\varrho)-\varepsilon$. Pick any continuous bounded function $\phi$ such that $\Phi_{\varrho}(\phi) \geqq D(\varrho)-\varepsilon / 2$. Then by (1.4),

$$
D\left(P_{t}^{*} \varrho\right) \geqq \Phi_{P_{t}^{*} \varrho}(\phi) \geqq \Phi_{\varrho}(\phi)-\|\phi\|_{\infty}\left\|P_{t}^{*} \varrho-\varrho\right\|_{L^{1}\left(\mathbf{R}^{n}, d^{n} x\right)} \geqq D(\varrho)-\varepsilon
$$

for any $t>0$ such that $\left\|P_{t}^{*} \varrho-\varrho\right\|_{L^{1}\left(\mathbf{R}^{n}, d^{n} x\right)} \leqq \varepsilon / 2\|\phi\|_{\infty}$. By the strong continuity of $\left\{P_{t}^{*} \mid t \geqq 0\right\}$, such a $t$ exists.

Next we note that since $\varrho \mapsto D(\varrho)$ is weakly lower semicontinuous, and $\left\{P_{t}^{*} \mid t \geqq 0\right\}$ is strongly continuous,

$$
S(\varrho) \geqq \lim _{t \rightarrow 0} S\left(P_{t}^{*} \varrho\right)
$$

so that in case $S(\varrho)=-\infty$, so does $\lim _{t \rightarrow 0} S\left(P_{t}^{*} \varrho\right)$.

This establishes continuity of $t \mapsto S\left(P_{t}^{*} \varrho\right)$ at $t=0$; clearly the same argument shows that it is continuous at all $t>0$ also. In fact, the second part is unnecessary for $t>0$ since by Lemma 1.1 it is clear that $S\left(P_{t}^{*} \varrho\right)>-\infty$.

To establish (1.22), note that by Jensen's inequality and (1.9),

$$
\begin{aligned}
S\left(P_{t}^{*} \varrho\right) & =S\left(e^{-t} X+\left(1-e^{-2 t}\right)^{1 / 2} G\right) \geqq S\left(\left(1-e^{-2 t}\right)^{1 / 2} G\right) \\
& =S(g)-\ln \left(1-e^{-2 t}\right)^{1 / 2} .
\end{aligned}
$$

We now turn to the final assertions of the lemma, so we suppose accordingly that $S(\varrho)>-\infty$. By the previous lemma, $P_{t}^{*} \varrho(x)$ is smooth in $x$ with all of its derivatives vanishing at infinity for all $t>0$. Using the formula (1.16) and Taylor's expansion with remainder to second order, it is easy to see that $t \mapsto P_{t}^{*} \varrho$ is strongly differentiable in $L^{1}\left(\mathbf{R}^{n}, d^{n} x\right)$, and that

where

$$
\frac{d}{d t} P_{t}^{*} \varrho=L\left(P_{t}^{*} \varrho\right)
$$

$$
L=\nabla \cdot(\nabla+x) \text {. }
$$

It is now a straightforward matter to show, using the regularity results of Lemma 1.1 , that $t \mapsto S\left(P_{t}^{*} \varrho\right)$ is continuously differentiable on $(0, \infty)$, and that $(1.23)$ and (1.24) hold. 
Lemma 1.3. Let $X_{1}, X_{2}, \ldots, X_{N}$ be independent random variables with $\sigma^{2}\left(X_{j}\right)=n$, $j=1, \ldots, N$. Let $0<\lambda_{1}, \lambda_{2}, \ldots, \lambda_{N}<1$ satisfy $\sum_{j=1}^{N} \lambda_{j}^{2}=1$. Then

$$
I\left(\sum_{j=1}^{N} \lambda_{j} X_{j}\right) \leqq \sum_{j=1}^{N} \lambda_{j}^{2} I\left(X_{j}\right)
$$

There is equality in (1.27) exactly when each $X_{j}$ has the same Gaussian density. If all the random variables are centered as well,

$$
J\left(\sum_{j=1}^{N} \lambda_{j} X_{j}\right) \leqq \sum_{j=1}^{N} \lambda_{j}^{2} J\left(X_{j}\right)
$$

and there is equality in (1.28) exactly when each $X_{j}$ has the density $g$.

Remarks. The inequality (1.27) is due to Stam [Sta]; his proof was later simplified by Blachman [Bla]. The proof sketched here is given in detail in [Car].

Proof. The assertions about $J$ clearly follow from those about $I$. Also it suffices to explicitly treat the case $N=2$ since the remaining cases follow by an obvious induction argument. We will only sketch a proof of this assertion since the details of the following proof can be found in [Car]. Suppose $X_{1}$ and $X_{2}$ have the densities $\varrho_{1}$ and $\varrho_{2}$ respectively.

The inequality

$$
\sum_{j=1}^{n} \int_{\mathbf{R}^{n}}\left(\frac{\partial}{\partial x_{j}}\left(\int_{\mathbf{R}^{n}}|f(x, y)|^{2} d^{n} y\right)^{1 / 2}\right)^{2} d^{n} x \leqq \sum_{j=1}^{n} \int_{\mathbf{R}^{2 n}}\left|\frac{\partial}{\partial x_{j}} f(x, y)\right|^{2} d^{n} x d^{n} y
$$

holds for all $f \in H^{1}\left(\mathbf{R}^{2 n}\right)$ where the derivatives are taken in the distributional sense, and when there is equality, $|f(x, y)|=\left|f_{1}(x)\right|\left|f_{2}(y)\right|$ for some $H^{1}\left(\mathbf{R}^{n}\right)$ functions $f_{1}$ and $f_{2}$. We apply this lemma by taking $f(x, y)=\varrho_{1}^{1 / 2}\left(\lambda_{1} x+\lambda_{2} y\right) \varrho_{1}^{1 / 2}\left(-\lambda_{2} x+\lambda_{1} y\right)$ and immediately obtain (1.27) on account of (1.10). There is equality only when

$$
\varrho_{1}\left(\lambda_{1} x+\lambda_{2} y\right) \varrho_{2}\left(-\lambda_{2} x+\lambda_{1} y\right)=\varrho_{3}(x) \varrho_{4}(y)
$$

for some densities $\varrho_{3}$ and $\varrho_{4}$. This implies [Kac, Ski, Car] that $\varrho_{1}$ and $\varrho_{2}$ are Gaussian with the same variance.

Theorem 1.1. Let $X_{1}, X_{2}, \ldots, X_{N}$ be independent random variables with $\sigma^{2}\left(X_{j}\right)=n$, $j=1, \ldots, N$. Let $0<\lambda_{1}, \lambda_{2}, \ldots, \lambda_{N}<1$ satisfy $\sum_{j=1}^{N} \lambda_{j}^{2}=1$. Then

$$
S\left(\sum_{j=1}^{N} \lambda_{j} X_{j}\right) \geqq \sum_{j=1}^{N} \lambda_{j}^{2} S\left(X_{j}\right) .
$$

Equality holds in (1.29) exactly when each $X_{j}$ has the same Gaussian density. Furthermore, for any $t>0$, define $\tilde{X}_{j}, j=1,2, \ldots, N$, by $\tilde{X}_{j}=e^{-t} X_{j}+\left(1-e^{-2 t}\right)^{1 / 2} G_{j}$. Then

$$
S\left(\sum_{j=1}^{N} \lambda_{j} \tilde{X}_{j}\right)-\sum_{j=1}^{N} \lambda_{j}^{2} S\left(\tilde{X}_{j}\right) \leqq S\left(\sum_{j=1}^{N} \lambda_{j} X_{j}\right)-\sum_{j=1}^{N} \lambda_{j}^{2} S\left(X_{j}\right) .
$$

Equality holds in (1.30) exactly when each $X_{j}$ has the same Gaussian density.

Remarks. Again, the inequality (1.29) is due to Stam [Sta] who used a heat semigroup approach different from our Ornstein-Uhlenbeck semigroup approach which leads directly to the stronger, and for us crucial, (1.30). 
Proof. If any $X_{N}$ fails to have a density, there is nothing to prove, so we may assume that $\sum_{j=1}^{N} \lambda_{j} X_{j}$ has the density $\varrho$. Notice that for all $s>0$, the density of $\sum_{j=1}^{N} \lambda_{j}\left(e^{-s} X_{j}+\left(1-e^{-2 s}\right)^{1 / 2} G_{j}\right)$ is $P_{s}^{*} \varrho$. Therefore by Lemma 1.2, then Lemma 1.3, and then Lemma 1.2 again,

$$
\begin{aligned}
S\left(\sum_{j=1}^{N} \lambda_{j} \tilde{X}_{j}\right) & =\int_{0}^{t} J\left(\sum_{j=1}^{N} \lambda_{j}\left(e^{-s} X_{j}+\left(1-e^{-2 s}\right)^{1 / 2} G_{j}\right)\right) d s+S\left(\sum_{j=1}^{N} \lambda_{j} X_{j}\right) \\
& \leqq \sum_{j=1}^{N} \lambda_{j}^{2} \int_{0}^{t} J\left(\left(e^{-s} X_{j}+\left(1-e^{-2 s}\right)^{1 / 2} G_{j}\right)\right) d s+S\left(\sum_{j=1}^{N} \lambda_{j} X_{j}\right) \\
& =\sum_{j=1}^{N} \lambda_{j}^{2}\left(S\left(\tilde{X}_{j}\right)-S\left(X_{j}\right)\right)+S\left(\sum_{j=1}^{N} \lambda_{j} X_{j}\right) .
\end{aligned}
$$

By the conditions for equality in Lemma 1.3 , there is equality here exactly when each $e^{-s} X_{j}+\left(1-e^{-2 s}\right)^{1 / 2} G_{j}$ has the density $g$, and this happens exactly when each $X_{j}$ itself has the density $g$. This establishes (1.30) and the statement concerning equality in it.

Thus unless $\varrho=g$, the left side of (1.30) - which implicitly depends on $t$ through the definition of the random variables $\widetilde{X}_{j}-$ is strictly decreasing as $t$ increases. By the first remark in this proof and (1.22), the limiting value is 0 . This establishes (1.29) and the statement concerning equality in it.

Throughout the rest of the paper, $\psi$ will always denote a positive continuous strictly decreasing function on $(0, \infty)$ with $\lim _{R \rightarrow \infty} \psi(R)=0$. For any density $\varrho$ with finite variance, $\psi_{\varrho}$ will always denote the function

$$
\psi_{\varrho}(R)=\int_{|x| \geqq R} x^{2} \varrho(x) d^{n} x .
$$

We adapt the notation to probability measures $\mu$ and random variables $X$ as usual and note that for any random variable $X$ we can write

$$
\psi_{X}(R)=E 1_{\{|X| \geqq R\}} X^{2} .
$$

Let $\mathscr{P}\left(\mathbf{R}^{n}\right)$ denote the set of Borel probability measures on $\mathbf{R}^{n}$.

Given a function $\psi$ as above and $t>0$ we define $\mathscr{K}_{\psi, t}$ by

$$
\mathscr{K}_{\psi, t}=\left\{\varrho \mid \varrho=P_{t}^{*} \mu, \mu \in \mathscr{P}\left(\mathbf{R}^{n}\right), \sigma^{2}(\mu)=n, m(\mu)=0, \psi_{\mu} \leqq \psi\right\}^{\mathrm{cl}},
$$

where the superscript cl denotes the closure in $L^{1}\left(\mathbf{R}^{n},\left(1+x^{2}\right) d^{n} x\right)$. We equip $\mathscr{K}_{\psi, t}$ with the relative topology. We will always assume that $\psi$ is such that $\mathscr{K}_{\psi, t}$ is not empty. Finally we denote by $\mathscr{B}_{t}$ the set of densities $\varrho$ which are of the form $P_{t}^{*} \mu$ for some $\mu \in \mathscr{P}\left(\mathbf{R}^{n}\right)$ which is centered with unit variance. This too is a subset of $L^{1}\left(\mathbf{R}^{n},\left(1+x^{2}\right) d^{n} x\right)$, and we equip it with the relative topology.

Lemma 1.4. Let $\psi$ and $t>0$ be as above be given. $\mathscr{K}_{\psi, t}$ is compact. Moreover, for any $0<\lambda<1$, the maps $\varrho \mapsto S(\varrho)$ and $\left(\varrho_{1}, \varrho_{2}\right) \mapsto \varrho_{1} *_{\lambda} \varrho_{2}$, respectively from $\mathscr{B}_{t}$ to $\mathbf{R}$ and $\mathscr{K}_{\psi, t}$ $\times \mathscr{K}_{\psi, t}$ to $\mathscr{B}_{t}$, are continuous.

Proof. Clearly $\mathscr{K}_{\psi, t}$ is bounded in $L^{1}\left(\mathbf{R}^{n},\left(1+x^{2}\right) d^{n} x\right)$. Given $\varepsilon>0$, choose $R$ so that $R^{-2}+\psi(R)<\varepsilon / 3$; clearly then for all $\varrho \in \mathscr{K}_{\psi, t}$,

$$
\int_{|x| \geqq R} \varrho(x)\left(1+x^{2}\right) d x \leqq \varepsilon / 3 .
$$


But by Lemma 1.1, $\mathscr{K}_{\psi, t}$ is uniformly equicontinuous on $\{|x| \leqq R\}$. Thus by the Arzela-Ascoli theorem we can select a finite $\varepsilon / 6\left(1+R^{2}\right)$ net in $\mathscr{C}(\{|x| \leqq R\})$ from among the restrictions of functions in $\mathscr{K}_{\psi, t}$ to $\{|x| \leqq R\}$. This procedure clearly leads to a finite $\varepsilon$ net in $\mathscr{K}_{\psi, t}$.

Next, suppose $\lim _{m \rightarrow \infty} \varrho_{m}=\varrho$ in $\mathscr{B}_{t}$. By Lemma 1.1, $|\ln \varrho(x)| \leqq A_{t}\left(1+x^{2}\right)$ for all $\varrho \in \mathscr{B}_{t}$ with $A_{t}$ depending only on $t$. Thus

$$
\left|S(\varrho)-S\left(\varrho_{m}\right)\right| \leqq 2 A_{t} \int_{\mathbf{R}^{n}}\left|\varrho(x)-\varrho_{m}(x)\right|\left(1+x^{2}\right) d^{n} x+\int_{\mathbf{R}^{n}} \varrho_{m}\left|\ln \varrho(x)-\ln \varrho_{m}(x)\right| d^{n} x,
$$

and the first term on the right clearly vanishes as $m$ increases. Suppose the second term on the right does not. Then there is an $\varepsilon>0$ so that, passing to a subsequence if need be,

and

$$
\int_{\mathbf{R}^{n}} \varrho_{m}(x)\left|\ln \varrho(x)-\ln \varrho_{m}(x)\right| d^{n} x \geqq \varepsilon \quad \forall m
$$

$$
\lim _{m \rightarrow \infty} \varrho_{m}(x)=\varrho(x) \quad \text { a.e. . }
$$

But $\varrho_{m}(x)\left|\ln \varrho(x)-\ln \varrho_{m}(x)\right| \leqq 2 A_{t}\left(1+x^{2}\right) \varrho_{m}(x)$, and the latter sequence of functions converges in $L^{1}\left(\mathbf{R}^{n}, d^{n} x\right)$ and is therefore uniformly integrable, and thus the first sequence is also. This together with the almost everywhere convergence above implies that

$$
\lim _{m \rightarrow \infty} \int_{\mathbf{R}^{n}} \varrho_{m}(x)\left|\ln \varrho_{m}(x)-\ln \varrho(x)\right| d^{n} x=0 .
$$

This contradicts the existence of a subsequence satisfying (1.34), and so $\varrho \mapsto S(\varrho)$ is continuous on $\mathscr{B}_{t}$.

Finally, $\varrho_{1} *_{\lambda} \varrho_{2}-\varrho_{3} *_{\lambda} \varrho_{4}=\left(\varrho_{1}-\varrho_{3}\right) *_{\lambda} \varrho_{2}+\varrho_{3} *_{\lambda}\left(\varrho_{2}-\varrho_{4}\right)$. Clearly we then have $\left\|\left(\varrho_{1}-\varrho_{3}\right) *_{\lambda} \varrho_{2}\right\|_{L^{1}\left(\mathbf{R}^{n}, d^{n} x\right)} \leqq\left\|\varrho_{1}-\varrho_{3}\right\|_{L^{1}\left(\mathbf{R}^{n}, d^{n} x\right)}$. Also

$$
\begin{aligned}
\int_{\mathbf{R}^{n}} x^{2}\left|\varrho_{1}-\varrho_{3}\right| *_{\lambda} \varrho_{2}(x) d^{n} x & =\frac{1}{\lambda\left(1-\lambda^{2}\right)^{1 / 2}} \int_{\mathbf{R}^{n}}\left|\varrho_{1}(x)-\varrho_{3}(x)\right||x+y|^{2} \varrho_{2}(y) d^{n} x d^{n} y \\
& \leqq \frac{2}{\lambda\left(1-\lambda^{2}\right)^{1 / 2}}\left\|\varrho_{1}-\varrho_{3}\right\|_{L^{1}\left(\mathbf{R}^{n},\left(1+x^{2}\right) d^{n} x\right)} .
\end{aligned}
$$

A similar argument applied to the other terms yields the result.

Theorem 1.2. Let $X_{1}$ and $X_{2}$ be independent random variables with zero mean and $\sigma^{2}\left(X_{1}\right)=\sigma^{2}\left(X_{2}\right)=n$. Suppose

$$
I\left(X_{1}\right), \quad I\left(X_{2}\right) \leqq I_{0}<\infty .
$$

Let $\psi$ be a given function on $[0, \infty)$ which decreases to zero at infinity such that $\psi_{X_{1}}$, $\psi_{X_{2}} \leqq \psi$. Also let some number a satisfying $0<a<\frac{1}{2}$ be given. Suppose for some $\varepsilon>0$,

$$
S\left(X_{1}\right) \leqq S(g)-\varepsilon .
$$

Then there is a $\delta_{\varepsilon}>0$ depending only on $I_{0}, \psi, a$ and $\varepsilon$ so that for any $\lambda$ with

$$
a \leqq \lambda^{2} \leqq 1-a
$$

such that $\left[\sigma\left(\lambda X_{1}+\left(1-\lambda^{2}\right)^{1 / 2} X_{2}\right)\right]=I_{n \times n}$, we have

$$
S\left(\lambda X_{1}+\left(1-\lambda^{2}\right)^{1 / 2} X_{2}\right)-\lambda^{2} S\left(X_{1}\right)-\left(1-\lambda^{2}\right) S\left(X_{2}\right) \geqq \delta_{\varepsilon} .
$$


Proof. Suppose first that the densities $\varrho_{1}$ and $\varrho_{2}$ of $X_{1}$ and $X_{2}$ both belong to $\mathscr{K}_{\psi, t}$ for some $t>0$.

By Lemma 1.4, the subset of $\mathscr{K}_{\psi, t}$ consisting of densities $\varrho$ with $D(\varrho) \geqq \varepsilon$ is closed and hence compact. Select a $\lambda_{1}$ satisfying (1.36); this determines $\lambda_{2}$. Thus with $\lambda_{1}$ fixed $S\left(\lambda_{1} X_{1}+\lambda_{2} X_{2}\right)-\lambda_{1}^{2} S\left(X_{1}\right)-\lambda_{2}^{2} S\left(X_{2}\right)$ depends only on $\varrho_{1}$ and $\varrho_{2}$. We will denote it by $\Delta_{\lambda_{1}}\left(\varrho_{1}, \varrho_{2}\right)$. By the continuity assertions of Lemma 1.4 it is a continuous function on $\mathscr{K}_{\psi, t} \times \mathscr{K}_{\psi, t}$. Therefore

$$
\inf \left\{\Delta_{\lambda_{1}}\left(\varrho_{1}, \varrho_{2}\right) \mid \varrho_{1}, \varrho_{2} \in \mathscr{K}_{\psi, t} D\left(\varrho_{1}\right) \geqq \varepsilon\right\}
$$

is attained at some pair $\left(\varrho_{\lambda_{1}}, \varrho_{\lambda_{2}}\right)$. We then define $\delta\left(\lambda_{1}\right)$ by

$$
\delta=\Delta_{\lambda_{1}}\left(\varrho_{\lambda_{1}}, \varrho_{\lambda_{2}}\right) .
$$

Since $\tilde{\varrho}_{1}$ is not $g$ on account of its positive relative entropy, $\delta_{\lambda_{1}}>0$ by the conditions for equality in Theorem 1.1 .

The result now follows once we have shown that $\lambda \mapsto \delta(\lambda)$ is continuous, for then $\inf \left\{\delta(\lambda) \mid a<\lambda^{2}<1-a\right\}$ is attained at some $\delta\left(\lambda_{a}\right)>0$; this is the lower bound $\delta_{\varepsilon}$ which we seek.

To establish this continuity, it clearly suffices to show that $\lambda \mapsto S\left(\lambda X_{1}\right.$ $\left.+\left(1-\lambda^{2}\right)^{1 / 2} X_{2}\right)$ is continuous. This is a simple consequence of (1.9), Jensen's inequality, and Theorem 1.1.

To treat the general case we first reguarlize as explained in the introduction, which amounts to applying $P_{t}^{*}$ to the densities $\varrho_{1}$ and $\varrho_{2}$. Clearly

$$
I\left(P_{t}^{*} \varrho_{1}\right), \quad I\left(P_{t}^{*} \varrho_{2}\right) \leqq I_{0} .
$$

Next, we have the elementary estimate

$$
\psi_{P_{t}^{*} \ell}(R) \leqq 2\left(\psi_{\varrho}(R / 2)+\psi_{g}(R / 2)\right) \quad \forall R, t>0 .
$$

Hence changing $\psi$ in a way that depends only on $\psi$, the regularized densities $P_{t}^{*} \varrho_{1}$ and $P_{t}^{*} \varrho_{2}$ belong to $\mathscr{K}_{\psi, t}$ with the modified $\psi$. Finally, it follows from (1.38) and Lemma 1.2 that for $t$ sufficiently small we have

$$
S\left(P_{t}^{*} \varrho_{1}\right) \leqq S(g)-\varepsilon / 2 .
$$

Thus the first part of the argument above applies uniformly to all regularized densities arising this way. By (1.30), the lower bound on the entropy production for the regularized densities is such that with it in place as $\delta_{\varepsilon},(1.37)$ is satisfied for the original unregularized random variables.

We shall make use of one more entropy and information inequality in what follows, namely:

$$
D(X) \leqq \frac{1}{2} J(X)
$$

for all centered random variables $X$ with unit variance. The inequality (1.39), due to Stam [Sta], is equivalent with Gross's logarithmic Sobolev inequality; see [Car]. To prove it, note that by (1.24) and (1.28),

$$
D(X)=\int_{0}^{\infty} J\left(e^{-t} X+\left(1-e^{-2 t}\right)^{1 / 2} G\right) d t \leqq \int_{0}^{\infty} e^{-2 t} J(X) d t .
$$




\section{Entropy and Central Limit Theorems}

We begin by establishing conditions under which block summation dynamics propagates uniform concentration in the sense discussed in the introduction. This result, together with the results of the last section enable us to easily prove several central limit theorems.

Let $\left\{X_{j} \mid j \in \mathbf{N}\right\}$ be a sequence of independent $\mathbf{R}^{n}$ valued random variables with non-degenerate covariance. We define two rectangular arrays $\left\{Y_{n, j} \mid n, j \in \mathbf{N}\right\}$ and $\left\{Z_{n, j} \mid n, j \in \mathbf{N}\right\}$ in terms of $\left\{X_{j} \mid j \in \mathbf{N}\right\}$ inductively as follows:

$$
\begin{array}{cl}
Y_{1, j}=X_{j}-m\left(X_{j}\right) & \forall j \in \mathbf{N}, \\
Y_{n, j}=Y_{n-1,2 j-1}+Y_{n-1,2 j} & n, j \in \mathbf{N}, n>1, \\
Z_{n, j}=\left[\sigma\left(X_{n, j}\right)\right]^{-1} Y_{n, j} & \forall n, j \in \mathbf{N} .
\end{array}
$$

It will be useful to introduce the notation

$$
[\sigma(n, j)]=\left[\sigma\left(X_{n, j}\right)\right] \text { and } \sigma(n, j)=\sigma\left(X_{n, j}\right) .
$$

For each $n$, let $\mathscr{Y}_{n}$ denote the sequence of independent random variables $\left\{Y_{n, j} \mid j \in \mathbf{N}\right\}$, and consider each $\mathscr{Y}_{n}$ as a process. In the same way, we introduce $\mathscr{Z}_{n}$. Finally, let $\mathscr{G}$ denote $\left\{G_{j} \mid j \in \mathbf{N}\right\}$, where the $G_{j}$ 's are independent mean zero, variance one random variables. Our object is to establish conditions under which

$$
\lim _{n \rightarrow \infty} \mathscr{Z}_{n}=\mathscr{G}
$$

in the sense of convergence of laws (as stochastic processes) in various topologies. The following result is formulated for real valued random variables, but it may be applied component by component for $\mathbf{R}^{d}$ valued random variables. We preface the theorem with a helpful observation.

Let $Z_{1}$ and $Z_{2}$ be independent mean zero, variance one random variables, and recall the notation $\psi_{Z}(R)=E 1_{\{|Z| \geqq R\}} Z^{2}$. Then since

$$
\begin{gathered}
\left(\left\{\left|Z_{1}\right| \geqq 2 R\right\} \cap\left\{\left|Z_{2}\right| \leqq R\right\}\right) \cup\left(\left\{\left|Z_{2}\right| \geqq 2 R\right\} \cap\left\{\left|Z_{1}\right| \leqq R\right\}\right) \subset\left\{\left|Z_{1}+Z_{2}\right| \geqq R\right\}, \\
\psi_{Z_{1}+Z_{2}}(R) \geqq \psi_{Z_{1}}(2 R) \operatorname{Pr}\left(\left\{\left|Z_{2}\right| \leqq R\right\}\right)+\psi_{Z_{2}}(2 R) \operatorname{Pr}\left(\left\{\left|Z_{1}\right| \leqq R\right\}\right) \\
\geqq \frac{1}{2}\left(\psi_{Z_{1}}(2 R)+\psi_{Z_{2}}(2 R)\right) \text { for all } R \geqq \sqrt{2} .
\end{gathered}
$$

This allows us to restrict our attention to sequences $\left\{X_{j} \mid j \in \mathbf{N}\right\}$ of even random variables in proving the next theorem because if $\left\{X_{j}^{\prime} \mid j \in \mathbf{N}\right\}$ is an independent copy of $\left\{X_{j} \mid j \in \mathbf{N}\right\}$, each $X_{j}-X_{j}^{\prime}$ is even.

Theorem 2.1. Let $\left\{X_{j} \mid j \in \mathbf{N}\right\}$ be a sequence of real valued random variables and derive $\mathscr{Y}_{n}$ and $\mathscr{Z}_{n}$ from it as above. Suppose there is a positive continuous function $\psi_{0}$ on $(0, \infty)$ decreasing to zero so that for all $R>0$ and all $j \in \mathbf{N}$,

$$
E 1_{\left\{\left|Z_{1, j}\right| \geqq R\right\}} Z_{i, j}^{2} \leqq \psi_{0}(R) .
$$

Suppose also that for some $a>0, \sigma^{2}(1, j) \geqq 1$ for all $j \in \mathbf{N}$.

For $\mathrm{L}>0$, define $\varphi_{L}$ by $\varphi_{L}(z)=z$ for $|z| \leqq L$, and $\varphi(z)=L \operatorname{sgn}(z)$ for $|z| \geqq L$. Put $\chi_{L}(z)=z-\varphi_{L}(z)$. We suppose further that

$$
0 \leqq \operatorname{cov}\left(\chi_{L}\left(Y_{n, j}\right), \chi_{L}\left(Y_{n, k}\right)\right) \leqq \operatorname{cov}\left(Y_{n, j}, Y_{n, k}\right)
$$

and that

$$
\lim _{n \rightarrow \infty} \sum_{j=1, j \neq k}^{\infty} \operatorname{cov}\left(Z_{n, j}, Z_{n, k}\right)=0
$$


uniformly in $k \in \mathbf{N}$. Finally suppose that

$$
E\left(\sum_{j=1}^{2^{p-n}} \varphi_{L \sigma\left(n, k 2^{p-n}+j\right)}\left(Y_{\left.n, k 2^{p-n}+j\right)}\right)\right)^{4} \leqq C L^{2} \sigma^{4}(p, k)
$$

for all $k, n$ and all $p>n$ and all $L>1$, where $C$ is a constant depending only on a.

Then there exists a function $\psi$ on $(0, \infty)$ decreasing to 0 so that

$$
E\left(1_{\left\{\left|Z_{n, j}\right| \geqq R\right\}} Z_{n, j}^{2}\right) \leqq \psi(R)
$$

for all $n, j \in \mathbf{N}$ and all $R>0$.

Remarks. In the independent case, the conditions (2.6) and (2.7) are trivially satisfied. To see that (2.8) is automatically satisfied in this case as well, first recall that we may assume each $Y_{n, j}$ is even so that $E \varphi_{L}\left(Y_{n, j}\right)=0$. Then

$$
\begin{aligned}
& E\left(\sum_{j=1}^{2 p-n} \varphi_{L \sigma\left(n, k 2^{p-n+j)}\right.}\left(Y_{n, k 2^{p-n}+j}\right)\right)^{4}=\sum_{j=1}^{2 p-n} E \varphi_{L \sigma\left(n, k 2^{p-n}+j\right)}^{4}\left(Y_{n, k 2^{p-n+j}}\right) \\
& +\sum_{j, m=1, j \neq m}^{2^{p-n}} E \varphi_{L \sigma\left(n, k 2^{p-n}+j\right)}^{2}\left(Y_{n, k 2^{p-n}+j}\right) E \varphi_{L \sigma\left(n, k 2^{p-n}+j\right)}^{2}\left(Y_{n, k 2^{p-n}+m}\right) \\
& \leqq\left(L^{2}+3\right) \sigma^{4}(p, k) \text {. }
\end{aligned}
$$

More generally, it is easy to see that since $\varphi_{L}$ and $\chi_{L}$ are both monotone, the condition (2.6) is satisfied when the underlying random variables satisfy the FKG inequalities. For background on these inequalities and the type of argument involved see [Sim, Ne80 and Ne83]. Condition (2.7) will often be easy to check in statistical mechanical applications as a consequence of finite "susceptibility." Condition (2.8) can be checked using the FKG inequalities and decay of the two point correlation function. All of this is discussed in the next section. In fact, it will be clear from the proof that (2.8) can be replaced by weaker conditions.

Proof. By the remark preceding the theorem, it suffices to treat the case in which all the $Y_{n, j}$ are even. Choose any $\varepsilon>0$. Then choose $n$ sufficiently large that $\sum_{j=1}^{\infty} E Z_{p, j} Z_{p, k}-1 \leqq \varepsilon / 2$ for all $p>n$. By symmetry, it suffices to establish the conclusion for $j=1$. Let $T$ be a positive number to be chosen later. Define $U_{j}$ $=\varphi_{T \sigma(n, j)}\left(Y_{n, j}\right)$ and $V_{j}=Y_{n, j}-U_{j}$. Also define $A_{R}=\left\{\left|Y_{p, 1}\right| \geqq R \sigma(n, 1)\right\}=\left\{\left|Z_{p, 1}\right| \geqq R\right\}$.

Then clearly for $p>n$,

$E 1_{A_{R}} Y_{p, 1}^{2}=E\left[\sum_{j=1}^{2^{p-n}} U_{j}+\sum_{j=1}^{2^{p-n}} V_{j}\right]^{2} \leqq 2 E 1_{A_{R}}\left[\sum_{j=1}^{2^{p-n}} U_{j}\right]^{2}+2 E\left[\sum_{j=1}^{2^{p-n}} V_{j}\right]^{2}$.

We first estimate the term on the left. By Chebychev's inequality, $\operatorname{Pr}\left(A_{R}\right) \leq R^{-2}$. By the Schwarz inequality and (2.8), $E 1_{A_{R}}\left[\sum_{j=1}^{2 p^{-n}} U_{j}\right]^{2} \leqq R^{-1}\left(E\left[\sum_{j=1}^{2^{p-n}} U_{j}\right]^{4}\right)^{1 / 2}$ $\leqq T R^{-1} C \sigma^{2}(p, 1)$, and so the first term in $(2.10)$ is no more than $\sigma^{2}(p, 1)(T / R)$.

Next, $E\left[\sum_{j=1}^{2 p-n} V_{j}\right]^{2}=\sum_{j=1}^{2^{p-n}} E V_{j}^{2}+\sum_{k=1}^{2^{p-n}} \sum_{j=1, j \neq k}^{2 p-n} E V_{j} V_{k}$. By the evenness assumption and (2.7)

$$
E V_{j} V_{k}=\operatorname{cov}\left(V_{j}, V_{k}\right) \leqq \operatorname{cov}\left(Y_{n, k}, Y_{n, j}\right) \leqq \frac{1}{2}\left(\sigma^{2}(n, j)+\sigma^{2}(n, k)\right) E Z_{n, j} Z_{n, k} .
$$


Also, since $n$ is fixed it readily follows from (2.6) that there exists a function $\psi_{n}$ decreasing to zero so that $\psi_{Z_{m, j}} \leqq \psi_{n}$ for all $j$ and all $m \leqq n$. We then have, using the fact that $\sum_{j=1} \sigma^{2}(n, j) \leqq \sigma^{2}(p, 1)$ on account of $(2.6)$,

$$
E\left[\sum_{j=1}^{2 p-n} V_{j}\right]^{2} \leqq \sum_{j=1}^{2 p-n} \sigma^{2}(n, j)\left[\psi_{Z_{n, j}}(T)+\varepsilon / 2\right] \leqq \sigma^{2}(p, 1)\left[\psi_{n}(T)+\varepsilon / 2\right]
$$

Combining these estimates and choosing $T=R^{1 / 2}$ yields

$$
\psi_{Z_{p, 1}}(R) \leqq C R^{-1 / 2}+\psi_{n}\left(R^{1 / 2}\right)+\psi_{n}(R)+\varepsilon / 2
$$

for all $R>0$ and all $p \in \mathbf{N}$. We can now choose an $R_{\varepsilon}$ independent of $p$ so that the sum of the first three terms above is less than $\varepsilon / 2$. Then $\psi_{z_{p, 1}}\left(R_{\varepsilon}\right) \leqq \varepsilon$ for all $p \in \mathbf{N}$.

Theorem 2.2. Let $\left\{X_{j} \mid j \in \mathbf{N}\right\}$ be a sequence of independent $\mathbf{R}^{d}$ valued random variables and derive $\mathscr{Y}_{n}$ and $\mathscr{Z}_{n}$ from it as above. Suppose there is a positive continuous function $\psi_{0}$ on $(0, \infty)$ decreasing to zero so that for all $R>0$ and all $j \in \mathbf{N}$,

$$
E 1_{\left\{\left|Z_{1, j}\right| \geqq R\right\}} Z_{1, j}^{2} \leqq \psi_{0}(R),
$$

and that for some $0<\sigma_{0}<\sigma_{1}<\infty$,

$$
\sigma_{0}^{2} \leqq \sigma^{2}\left(X_{j}\right) \leqq \sigma_{1}^{2} \quad \forall j \in \mathbf{N} .
$$

In the multivariate case, assume moreover that

$$
\lim _{n \rightarrow \infty} \sup \left\{\sigma^{-1}(n, j)\left\|[\sigma(n, j)]-\frac{\sigma(n, j)}{d} I_{n \times n}\right\| \mid j \in \mathbf{N}\right\}=0,
$$

where $\|\cdot\|$ denotes, say, the Hilbert-Schmidt norm. Suppose also that for some $I_{0}<\infty$,

Then

$$
I\left(Z_{1, j}\right) \leqq I_{0} \quad \forall j \in \mathbf{N}
$$

in the sense that

$$
\lim _{n \rightarrow \infty} \mathscr{Z}_{n}=\mathscr{G}
$$

$$
\lim _{n \rightarrow \infty} \sup \left\{D\left(Z_{n, j}\right) \mid j \in \mathbf{N}\right\}=0 .
$$

In particular, $\lim _{n \rightarrow \infty} D\left(\sum_{j=1}^{n} Y_{1, j} / \sigma\left(\sum_{j=1}^{n} Y_{1, j}\right)\right)=0$.

Proof. We will first separately treat the case $d=1$. Note that by the definitions (2.2), (2.3) and (2.4), $Z_{n+1, j}=\lambda_{n, j} Z_{n, 2 j-1}+\left(1-\lambda_{n, j}\right)^{1 / 2} Z_{n, 2 j}$, where

$$
\lambda_{n, j}^{2}=\frac{\sigma^{2}(n, 2 j-1)}{\sigma^{2}(n, 2 j-1)+\sigma^{2}(n, 2 j)}=\frac{\sigma^{2}(n, 2 j-1)}{\sigma^{2}(n+1, j)} .
$$

By (2.12) it follows that for some $0<a<1 / 2$,

$$
a \leqq \lambda_{n, j}^{2} \leqq 1-a \quad \forall n, j \in \mathbf{N} .
$$

By Lemma 2.1, $\psi_{Z_{n}, j}(R) \leqq \psi(R)$ with $\psi$ and $\psi_{0}$ decreasing to zero. Finally, by (1.27), $I\left(Z_{n, j}\right) \leqq I_{0}$ for all $n, j \in \mathbf{N}$. Thus it follows from Theorem 1.2 that either

$$
S\left(Z_{n+1, j}\right)-\lambda_{n, j}^{2} S\left(Z_{n, 2 j-1}\right)-\left(1-\lambda_{n, j}^{2}\right) S\left(Z_{n, 2 j}\right) \geqq \delta_{\varepsilon}
$$


for some $\delta_{\varepsilon}>0$ independent of $n$ and $j$, or

$$
S\left(Z_{n, 2 j-1}\right) \geqq S(g)-\varepsilon \quad \text { and } \quad S\left(Z_{n, 2 j}\right) \geqq S(g)-\varepsilon .
$$

Now define $s_{n}$ by $s_{n}=\inf \left\{S\left(Z_{n, j}\right) \mid j \in \mathbf{N}\right\}$. Notice that by (1.39),

$$
s_{1} \geqq S_{0}=S(g)-\frac{1}{2}\left(1-I_{0}\right) \text {. }
$$

We now claim that

$$
s_{n+1} \geqq(S(g)-\varepsilon) \wedge\left(s_{n}+\delta_{\varepsilon}\right) .
$$

To see this, note that if (2.18) holds, $S\left(Z_{n+1, j}\right) \geqq S_{n}+\delta_{\varepsilon}$ by the definition of $s_{n}$. But in case (2.19) holds, then by Shannon's inequality, $S\left(Z_{n+1, j}\right) \geqq S(g)-\varepsilon$. Thus $S\left(Z_{n+1, j}\right)$ $\geqq\left(s_{n}+\delta_{\varepsilon}\right) \wedge(S(g)-\varepsilon)$ for every $j$, and (2.20) holds.

Since $S_{n+1} \geqq S_{n}$ by Shannon's inequality, it clearly follows that $\lim _{n \rightarrow \infty} s_{n}=S(g)$; that is, (2.14) holds.

To recover a central limit theorem in the standard form - but with a very strong sense of convergence - we again fix an $\varepsilon>0$, and then choose a $k$ so large that $s_{k}$ $\geqq S(g)-\varepsilon$. By the above argument, such a $k$ exists. Now with this $k$ fixed, any $n$ can be uniquely expressed as $n=m 2^{k}+r, 0 \leqq r<2^{k}$. Define $Y=\sum_{j=m 2^{k}+1}^{n} Y_{1, j}$ and
$Z=Y / \sigma(Y)$. Then

$$
\sum_{j=1}^{n} Y_{1, j} / \sigma(1, n)=\sum_{q=1}^{m-1} \frac{\sigma(k, q)}{\sigma(1, n)} Z_{k, q}+\frac{\sigma(Y)}{\sigma(1, n)} Z
$$

so that again by Theorem 1.1,

$$
\begin{aligned}
S\left(\sum_{j=1}^{n} Y_{1, j} / \sigma(1, n)\right) & \geqq \sum_{q=1}^{m-1} \frac{\sigma^{2}(k, q)}{\sigma^{2}(1, n)} S\left(Z_{k, q}\right)+\frac{\sigma^{2}(Y)}{\sigma^{2}(1, n)} S(Z) \\
& \geqq\left(1-\frac{\sigma^{2}(Y)}{\sigma^{2}(1, n)}\right)(S(g)-\varepsilon)+\frac{\sigma^{2}(Y)}{\sigma^{2}(1, n)} S_{0} \geqq S(g)-2 \varepsilon
\end{aligned}
$$

for all $n$ sufficiently large since $\lim _{n \rightarrow \infty} \sigma(1, n)=\infty$ by $(2.12)$.

To handle the multivariate case, introduce the matrices

$$
\begin{gathered}
A_{n, j}=\frac{\sigma(n+1, j)}{\sigma(n, 2 j-1)}[\sigma(n+1, j)]^{-1}[\sigma(n, 2 j-1)], \\
B_{n, j}=\frac{\sigma(n+1, j)}{\sigma(n, 2 j)}[\sigma(n+1, j)]^{-1}[\sigma(n, 2 j)],
\end{gathered}
$$

and the random variables

$$
W_{n, 2 j-1}=A_{n, j} Z_{n, 2 j-1}, \quad W_{n, 2 j}=B_{n, j} Z_{n, 2 j} .
$$

Then with $\lambda_{n, j}$ still defined by (2.16),

$$
Z_{n+1, j}=\lambda_{n, j} W_{n, 2 j-1}+\left(1-\lambda_{n, j}^{2}\right)^{1 / 2} W_{n, 2 j}
$$

and $\sigma^{2}\left(W_{n, j}\right)=d$. Next define $r_{n}=\sup \left\{\left|S\left(W_{n, j}\right)-S\left(Z_{n, j}\right)\right| \mid j \in \mathbf{N}\right\}$ and note that on account of the scaling property (1.9) of the entropy, and our additional assumption under (2.10), $\lim _{n \rightarrow \infty} r_{n}=0$.

The reasoning used above then yields $s_{n} \geqq\left(S(g)-\varepsilon-r_{n}\right) \wedge\left(s_{n}+\delta_{\varepsilon}-r_{n}\right)$, and the rest of the proof proceeds straightforwardly. 
Remarks. In the identically distributed case, (2.11) and (2.12) hold as soon as $X_{1}$ has finite variance. Assume that $X_{1}$ itself has zero mean and unit variance, and that, instead of (2.13), $S\left(X_{1}\right)>-\infty$. Then by (1.30), using the notation of Theorem 1.1,

$$
S\left(\frac{1}{\sqrt{N}} \sum_{j=1}^{N} X_{j}\right) \geqq S\left(\frac{1}{\sqrt{N}} \sum_{j=1}^{N} \tilde{X}_{j}\right)+\left(S\left(\tilde{X}_{1}\right)-S\left(X_{1}\right)\right)
$$

for any choice of $t$ in the regularization defining $\tilde{X}_{j}$. Choosing $t$ sufficiently small, the last term on the right can be made arbitrarily small by Lemma 1.2. Clearly $I\left(\tilde{X}_{1}\right)<\infty$, so that $\lim _{N \rightarrow \infty} S\left(\frac{1}{\sqrt{N}} \sum_{j=1}^{N} \tilde{X}_{j}\right)=S(g)$, so that also $\lim _{N \rightarrow \infty} S\left(\frac{1}{\sqrt{N}} \sum_{j=1}^{N} X_{j}\right)$ $=S(g)$. Thus in the i.i.d. case, the condition (2.13) can be relaxed to $S\left(X_{1}\right)>-\infty$, and our result strictly generalizes that of Barron [Bar].

Note that (2.11) is implied by the Lindeberg condition; however, note that the Lindeberg condition does not imply (2.14) even in distribution.

We now turn to dependent random variables.

Theorem 2.3. Let $\left\{X_{j} \mid j \in \mathbf{N}\right\}$ be a sequence of $\mathbf{R}^{d}$ valued random variables satisfying the hypotheses of Theorem 2.1, and derive $\mathscr{Y}_{n}$ and $\mathscr{Z}_{n}$ from it as above. For each $t>0$, define $\mathscr{Z}$ by $\tilde{Z}_{n, j}=e^{-t} Z_{n, j}+\left(1-e^{-2 t}\right)^{1 / 2} G_{n, j}$. Suppose that there is an array $\left\{\lambda_{n, j} \mid n, j \in \mathbf{N}\right\}$ of numbers with

$$
a \leqq \lambda_{n, 1}^{2} \leqq 1-a
$$

for all $n$ and $j$ and some fixed $a>0$ such that for all $t>0$

$\lim _{n \rightarrow \infty} \sup \left\{\left[S\left(\lambda_{n, j} \tilde{Z}_{n, 2 j-1}+\left(1-\lambda_{n, j}^{2}\right)^{1 / 2} \tilde{W}_{n, 2 j}\right)-S\left(\tilde{Z}_{n+1, j}\right)\right]_{+} \mid j \in \mathbf{N}\right\}=0$,

where $W_{n, 2 j}$ is an independent copy of $Z_{n, 2 j}$ and $[\cdot]_{+}$denotes the positive part. Then, letting $\mu_{n, j}$ denote the law of $Z_{n, j}$ for all Borel sets $A$,

$$
\lim _{n \rightarrow \infty} \mu_{n, j}(A)=\int_{A} g(x) d^{d} x
$$

Proof. Select any $t>0$ and define the densities $\varrho_{n, j}$ by $\varrho_{n, j}=P_{t}^{*} \mu_{n, j}$ for all $n, j \in \mathbf{N}$. Also define $\xi_{n, j}=\left|S\left(\varrho_{n, 2 j-1} *_{\lambda_{n, j}} \varrho_{n, 2 j}\right)-S\left(\varrho_{n+1, j}\right)\right|$ and $\xi_{n}=\sup \left\{\xi_{n, j} \mid j \in \mathbf{N}\right\}$.

Select any $\varepsilon>0$. By Theorem 2.1, (2.21) and the regularization, the hypotheses of Theorem 1.2 are satisfied uniformly in $n$ and $j$. Thus by Theorem 1.2, there is a $\delta_{\varepsilon}>0$ depending only $t, a, \varepsilon$ and the $\psi$ supplied by Theorem 2.1 so that either

$$
S\left(\varrho_{n, 2 j-1} *_{\lambda_{n, j}} \varrho_{n, 2 j}\right)-\lambda_{n, j}^{2} S\left(\varrho_{n, 2 j-1}\right)-\left(1-\lambda_{n, j}^{2}\right) S\left(\varrho_{n, 2 j}\right)>\delta_{\varepsilon}
$$

or both of $S\left(\varrho_{n, 2 j-1}\right)$ or $S\left(\varrho_{n, 2 j}\right)$ exceed $S(g)-\varepsilon$. By (2.22) there is an $N_{\varepsilon}$ so that $\xi_{n}<\delta_{\varepsilon} / 2$ for all $n \geqq N_{\varepsilon}$. Note that we must have $\delta_{\varepsilon}<\varepsilon$, and that on account of the regularization and (1.39), $S\left(\varrho_{n, j}\right) \geqq S_{0}>-\infty$ for all $n$ and $j$.

Now, as in the proof of the last theorem, let $s_{n}=\inf \left\{S\left(\varrho_{n, j}\right) \mid j \in \mathbf{N}\right\}$. Then for all $n \geqq N_{\varepsilon}$ and all $j$,

$$
\begin{aligned}
S\left(\varrho_{n+1, j}\right) & \geqq S\left(\varrho_{n, 2 j-1} *_{\lambda_{n, 3}} \varrho_{n, 2 j}\right)-\xi_{n} \\
& \geqq\left(s_{n}+\delta_{\varepsilon}\right) \wedge(S(g)-\varepsilon)-\delta_{\varepsilon} / 2 \geqq\left(s_{n}+\delta_{\varepsilon} / 2\right) \wedge(S(g)-3 \varepsilon / 2) .
\end{aligned}
$$

As before, this clearly implies $\lim _{n \rightarrow \infty} s_{n}=S(g)$. 
Finally, by the Kullback-Liebler inequality [Kul], $\left\|\varrho_{n, j}-\mathrm{g}\right\|_{L^{1}\left(\mathbf{R}_{d}, d^{d} x\right)}^{2} \leqq 2 D\left(\varrho_{n, j}\right)$, so that $\lim _{n \rightarrow \infty}\left\|P_{t}^{*} \mu_{n, j}-g\right\|_{L^{1}\left(\mathbf{R}^{d}, d^{d} x\right)}=0$. Thus for any function $h \in \mathscr{C}_{0}\left(\mathbf{R}^{d}\right)$,

$$
\begin{aligned}
& \lim _{n \rightarrow \infty}\left|\int_{\mathbf{R}^{d}} h(x)\left(d \mu_{n, j}(x)-g(x) d^{d} x\right)\right| \\
& \quad \leqq \lim _{n \rightarrow \infty}\left|\int_{\mathbf{R}^{d}}\left(P_{t} h(x)-h(x)\right) d \mu_{n, 1}(x)\right|+\lim _{n \rightarrow \infty}\left|\int_{\mathbf{R}^{d}} P_{t} h(x)\left(d \mu_{n, 1}(x)-g(x) d^{d} x\right)\right| \\
& \quad \leqq 2\left\|P_{t} h-h\right\|_{\mathscr{C}_{0}\left(\mathbf{R}^{d}\right)}+\lim _{n \rightarrow \infty}\|h\|_{\mathscr{C}_{0}\left(\mathbf{R}^{d}\right)}\left\|P_{t}^{*} \mu_{n, 1}-g\right\|_{L^{d}\left(\mathbf{R}^{d}\right)},
\end{aligned}
$$

where we have used the fact that $P_{t}^{*} g=g$. From the strong continuity of $\left\{P_{t} \mid t \geqq 0\right\}$ on $\mathscr{C}_{0}\left(\mathbf{R}^{d}\right)$, we then obtain our result.

Theorem 2.4. Let $\left\{X_{j} \mid j \in \mathbf{N}\right\}$ be a sequence of $\mathbf{R}^{d}$ valued random variables satisfying the hypotheses of Theorem 2.1, and derive $\mathscr{Y}_{n}$ and $\mathscr{Z}_{n}$ from it as above. Suppose that there is an array $\left\{\lambda_{n, j} \mid n, j \in \mathbf{N}\right\}$ of numbers satisfying (2.21) such that uniformly for $y$ in an arbitrary compact set

$$
\lim _{n \rightarrow \infty} \sup \left\{\left|\phi_{Z_{n+1, j}}(y)-\phi_{Z_{n, 2 j-1}}\left(\lambda_{n, j} y\right) \phi_{Z_{n, 2 j}}\left(\left(1-\lambda_{n, j}^{2}\right)^{1 / 2} y\right)\right| \mid j \in \mathbf{N}\right\}=0,
$$

where $\phi_{X}(y)$ denotes the characteristic function $E e^{i y \cdot X}$. Then the hypotheses of Theorem 2.3 are satisfied. If moreover for each $N \in \mathbf{N}$,

$$
\lim _{n \rightarrow \infty} \sup \left\{\left|\phi_{k=1}^{N} Z_{n, j_{k}}^{N}(y)-\prod_{k=1}^{N} \phi_{Z_{n, j_{k}}}(y)\right| \mid j_{1} \neq \ldots \neq j_{N} \in \mathbf{N}\right\}=0 .
$$

as above, then $\lim _{n \rightarrow \infty} \mathscr{Z}_{n}=\mathscr{G}$ in distribution.

Proof. We use the notation established in the proof of Theorem 2.3. Our first goal is to establish that $\lim _{n \rightarrow \infty} \xi_{n}=0$.

Suppose this is not the case; then we can find an $\varepsilon>0$ and subsequences $\left\{n_{k} \mid k \in \mathbf{N}\right\}$ and $\left\{j_{k} \mid k \in \mathbf{N}\right\}$ of the integers such that

$$
\xi_{n_{k}, j_{k}} \geqq \varepsilon \quad \forall k \in \mathbf{N} .
$$

But on account of Theorem 2.1, the regularization, and Lemma 1.4, the set of densities $\left\{\varrho_{n, j} \mid \mathrm{n}, \mathrm{j} \in \mathbf{N}\right\}$ has compact closure in $L^{1}\left(\mathbf{R}^{d},\left(1+x^{2}\right) d^{d} x\right)$. Thus passing to further subsequences as necessary, we can assume that for densities $\varrho_{a}, \varrho_{b}$ and $\varrho_{c}$,

$\lim _{k \rightarrow \infty} \varrho_{n_{k}, 2 j_{k}-1}=\varrho_{a}, \quad \lim _{k \rightarrow \infty} \varrho_{n_{k}, 2 j_{k}}=\varrho_{b} \quad$ and $\quad \lim _{k \rightarrow \infty} \varrho_{n_{k}+1, j_{k}}=\varrho_{c}$,

where all the limits hold both almost everywhere and in $L^{1}\left(\mathbf{R}^{d},\left(1+x^{2}\right) d^{d} x\right)$. Passing to a further subsequence as necessary, we may also assume that $\lim _{k \rightarrow \infty} \lambda_{n_{k}, j_{k}}=\lambda$
exists; clearly $a<\lambda^{2}<1-a$.

We then have

$$
\begin{gathered}
\lim _{k \rightarrow \infty} \phi \varrho_{n_{k}, 2 j_{k}-1}(y)=\phi \varrho_{a}(y), \\
\lim _{k \rightarrow \infty} \phi \varrho_{n_{k}, 2 j_{k}}(y)=\phi \varrho_{b}(y) \text { and } \lim _{k \rightarrow \infty} \phi \varrho_{n_{k}+1, j}(y)=\phi \varrho_{c}(y)
\end{gathered}
$$

for all $y$. We conclude from this and (2.23) that $\varrho_{c}=\varrho_{a} *_{\lambda} \varrho_{b}$ and thus that

$$
S\left(\varrho_{a} *_{\lambda} \varrho_{b}\right)=S\left(\varrho_{c}\right) \text {. }
$$


Now by Lemma 1.1, $\left|\ln \varrho_{n_{k}+1, j}(x)\right| \leqq A\left(1+x^{2}\right)$ for some $A$ independent of $k$ and $j$. This together with (2.26) implies

$$
\lim _{k \rightarrow \infty} S\left(\varrho_{n_{k}+1, j}\right)=S\left(\varrho_{c}\right)
$$

by an argument employed in the proof of Lemma 1.4.

Similarly one sees that

$$
\lim _{k \rightarrow \infty} S\left(\varrho_{n_{k}, 2 j_{k}-1} *_{\lambda_{n_{k}}, j_{k}} \varrho_{n_{k}, 2 j_{k}}\right)=S\left(\varrho_{a} *_{\lambda} \varrho_{b}\right) .
$$

Combining (2.27), (2.28) and (2.29) we see that no subsequence satisfying (2.25) exists. This establishes that (2.22) of Theorem 2.3 holds. Then from (2.24) and Theorem 2.3, the final conclusion readily follows.

In treating the ferromagnetic systems we discuss in the next section, it is sometimes useful to work with a version of this result stated in terms of moment generating functions. In such a setting one often has exponential moment inequalities which make Theorem 2.1 superfluous; see [Ne75a] and Lemma 3.2 of the next section.

Recall that for $\mu \in \mathscr{P}(\mathbf{R})$, its logarithmic moment generating function $\Lambda_{\mu}$ is defined by

$$
\Lambda_{\mu}(y)=\ln \left(\int_{\mathbf{R}} e^{x y} d \mu(x)\right)
$$

As usual, in case $X$ is a random variable with law $\mu$, we may write $\Lambda_{X}$ for $\Lambda_{\mu}$.

It is useful for applications in statistical mechanics to have a version of the last theorem stated in terms of moment generating functions instead of characteristic functions. This entails only trivial changes in the proof, so we state the following result without proof:

Theorem 2.5. Let $\left\{X_{j} \mid j \in \mathbf{N}\right\}$ be a sequence of $\mathbf{R}^{d}$ valued random variables and derive $\mathscr{Y}_{n}$ and $\mathscr{Z}_{n}$ from it as above. Suppose that for some $b>0, \Lambda_{Z_{n, j}}(y) \leqq 1$ for all $y$ with $y_{i} \in(0, b)$ for $i=1, \ldots, d$ for all $n$ and $j$. Suppose also that there is an array $\left\{\lambda_{n, j} \mid n, j \in \mathbf{N}\right\}$ of numbers satisfying (2.21) such that uniformly for $y$ in the set described above

$\lim _{n \rightarrow \infty} \sup \left\{\left|\Lambda_{Z_{n+1, j}}(y)-\Lambda_{Z_{n, 2 j-1}}\left(\lambda_{n, j} y\right)-\Lambda_{Z_{n, 2 j}}\left(\left(1-\lambda_{n, j}^{2}\right)^{1 / 2} y\right)\right| \mid j \in \mathbf{N}\right\}=0$.

Then the hypotheses of Theorem 2.3 are satisfied. If moreover for each $N \in \mathbf{N}$,

$$
\lim _{n \rightarrow \infty} \sup \left\{\left|\Lambda \sum_{k=1}^{N} Z_{n, j_{k}}(y)-\prod_{k=1}^{N} \Lambda Z_{n, j_{k}}(y)\right| \mid j_{1} \neq \ldots \neq j_{N} \in \mathbf{N}\right\}=0
$$

as above, then $\lim _{n \rightarrow \infty} \mathscr{Z}_{n}=\mathscr{G}$ in distribution.

\section{Central Limit Theorems for Extensive Variables Associated with Certain Spin Systems}

In this section we introduce a class of lattice spin systems and some associated extensive random variables, and then prove central limit theorems for them.

Let $\mathscr{S}$ be a compact subset of $\mathbf{R}^{D}$. Let $r(\mathscr{S})=\sup \{|s| \mid s \in \mathscr{S}\} . \mathscr{S}$ will often be a discrete set of points, but to avoid triviality, with $r(\mathscr{S})>0$. For a region $V$ in $\mathbf{Z}^{d}$, the 
spin configuration space $\Omega_{V}$ is $\mathscr{S}^{V}$; that is $\Omega_{V}$ consists of all maps $\omega: \mathrm{V} \rightarrow \mathscr{S}$, and is equipped with the product topology. For each $i \in V$ the spin variable $S_{i}$ is defined by $S_{i}(\omega)=\omega(i)$.

We take as given data a field of bilinear forms $J_{i, j}$ on $\mathbf{R}^{D} \times \mathbf{R}^{D},(i, j) \in \mathbf{Z}^{d} \times \mathbf{Z}^{d}$ called couplings, and a field of vectors $h_{i}$ in $\mathbf{R}^{D}, i \in \mathbf{Z}^{d}$, called the applied field. We write $|J|$ to denote

$$
\sup \left\{J_{i, j}\left(s_{1}, s_{2}\right) /\left|s_{1}\right|\left|s_{2}\right| \mid(i, j) \in \mathbf{Z}^{d} \times \mathbf{Z}^{d}, s_{1}, s_{2} \in \mathbf{R}^{D}\right\} ;
$$

we write $|h|$ to denote $\sup \left\{\left|h_{i}\right| \mid i \in \mathbf{Z}^{d}\right\}$; and we assume $|J|$ and $|h|$ are finite.

For any region $V \subset \mathbf{R}^{D}$, we define a Hamiltonian $H_{V}$ by

$$
H_{V}(\omega)=-\sum_{i, j \in V} J_{i, j}\left(S_{i}(\omega), S_{j}(\omega)\right)-\sum_{i \in V} h_{i} \cdot S_{i}(\omega),
$$

where the centered dot denotes the Euclidean inner product in $\mathbf{R}^{D}$.

We suppose for convenience that the coupling $J_{i, j}$ vanishes unless $|i-j|=1$. With a little more work, rapidly decaying couplings could certainly be treated by the methods that follow, though exactly how much decay is required is an interesting question. Here for $i=\left(n_{1}, \ldots, n_{d}\right) \in \mathbf{Z}^{d}$, $|i|$ denotes $\left|n_{1}\right|+\ldots+\left|n_{d}\right|$. For $V \subset \mathbf{Z}^{d},|V|$ denotes the number of lattice points in $V$. For $V_{1}, V_{2} \subset \mathbf{Z}^{d}$, the distance between them is denoted $d\left(V_{1}, V_{2}\right)$. Finally, $\partial V$ denotes the set $\{i \in V|| i-j \mid=1$ for some $j \notin V\}$.

The applied field is called non-negative in case all of the components - in some fixed orthonormal basis - of each $h_{i}$ are non-negative. The couplings are called ferromagnetic in case each $J_{i j}$ is a positive form. The spin system is called ferromagnetic in case the couplings are ferromagnetic and the applied field is nonnegative.

Now fix a reference probability measure $\mu$ on $\mathbf{R}^{D}$ which has $\mathscr{S}$ for its support. Let $\mu_{i}$ be a copy of $\mu$ for each $i \in \mathbf{Z}^{d}$, and for any region $V$ define a reference probability measure $v_{V}$ on $\Omega_{V}$ by $v_{V}=\prod_{i \in V} \mu_{i}$. In what follows, we suppose that $\mu$ is invariant under each of the reflections

$$
R_{m}:\left(s^{1}, \ldots, s^{m}, \ldots, s^{D}\right) \mapsto\left(s^{1}, \ldots,-s^{m}, \ldots, s^{D}\right) .
$$

Of course, this implies that $\mathscr{S}$ itself possesses the corresponding symmetries.

For fixed inverse temperature $\beta$, - suppressed in our notation - we define probability measures $\operatorname{Pr}_{V}$ on the sample spaces $\Omega_{V}$ by

$$
\int_{\Omega_{V}} F(\omega) d P r_{V}(\omega)=Z(V)^{-1} \int_{\Omega_{V}} F(\omega) e^{-\beta H_{V}(\omega)} d v_{V}(\omega)
$$

for all continuous functions $F$ on $\Omega_{V} ; Z(V)=\int_{\Omega_{V}} e^{-\beta H_{V}(\omega)} d v_{V}(\omega)$. We use the standard notation $\langle F\rangle_{V}=\int_{\Omega_{V}} F(\omega) d \operatorname{Pr}_{V}(\omega)$ for thermal averages. We denote the covariance matrix of any $\mathbf{R}^{m}$ valued random variable $F$ on $\Omega_{V}$ under $\operatorname{Pr}_{V}$ by $\left[\sigma_{V}(F)\right]^{2}$, and we denote its variance by $\sigma_{V}^{2}(F)$.

The magnetization $M_{V}$ in the region $V$ is the random variable $M_{V}(\omega)$ $=\sum_{i \in V} S_{i}(\omega)$. We denote its covariance matrix simply by $\left[\sigma_{V}\right]^{2}$, and its variance by $\sigma_{V}^{2}$. The normalized magnetization $m_{V}$ is

$$
m_{V}(\omega)=\left[\sigma_{V}\right]^{-1}\left(M_{V}(\omega)-\left\langle M_{V}\right\rangle_{V}\right) .
$$


We will examine the limiting behavior of the law of $m_{V_{n}}$ for sequences of regions $V_{n}$ arising in a block spin summation procedure as follows:

Let $\left\{e_{1}, \ldots, e_{d}\right\}$ denote the orthonormal basis of $\mathbf{R}^{d}$ generating $\mathbf{Z}^{d}$. We proceed to define $\left\{V_{n, j} \mid j \in \mathbf{N}\right\}$ inductively. First pick any square block containing the origin, and call it $V_{1,1}$. For any $n \in \mathbf{N}$, write $n=p 2 d+r, 0 \leqq r<2 d$. Let $V_{n, 2}$ be the reflection of $V_{n, 1}$ about the bounding plane of $V_{n, 1}$ possessing $e_{r+1}$ as its outward normal in case $0 \leqq r<d$, and possessing $-e_{r+1-d}$ as its outward normal in case $d \leqq r<2 d$. We then put $V_{n+1,1}=V_{n, 1} \cup V_{n, 2}$. Finally, for each $n$, we cover $\mathbf{Z}^{d}$ by translates of $V_{n, 1}$ so that the boundaries overlap as above, and we enumerate the blocks so that $V_{n+1, j}$ $=V_{n, 2 j-1} \cup V_{n, 2 j}$ for all $n$ and $j$. Note that the volume doubling procedure we use is balanced so that we obtain $\bigcup_{n \in \mathbf{N}} V_{n, 1}=\mathbf{Z}^{d}$.

We verify the hypotheses of Theorem 2.2 in this context by estimating the effect on the distribution of $m_{V_{n, j}}$ of decoupling the spins in $V_{n-1,2 j-1}$ from those in $V_{n-1,2 j}$.

Define $B_{n, j}$ by

$$
B_{n, j}=\left\{i \in V_{n, j} \mid d\left(\{i\}, V_{n-1,2 j-1} \cap V_{n-1,2 j}\right) \leqq 1\right\} \cup \partial V_{n, j} .
$$

To avoid an excessive proliferation of indices, we will focus on $j=1$, and will write $B_{n}$ for $B_{n, 1}, V_{n}$ for $V_{n, 1}$, and $U_{n}$ for $V_{n, 2}$. For $\lambda \in[0,1]$, define a Hamiltonian $H_{V_{n} ; \lambda}$ by

$$
H_{V_{n} ; \lambda}(\omega)=H_{V_{n}}(\omega)-(1-\lambda) \sum_{i, j \in B_{n}} J_{i, j}\left(S_{i}(\omega), S_{j}(\omega)\right) \text {. }
$$

For given $\beta>0$ define probability measures $\operatorname{Pr}_{V_{n} ; \lambda}$ in terms of $H_{V_{n} ; \lambda}$ as above. Denote thermal averages with respect to these probability measures by $\langle\cdot\rangle_{V_{n}} ; \lambda$. We denote the variance of the magnetization under $\operatorname{Pr}_{V_{n} ; \lambda}$ by $\sigma_{V_{n} ; \lambda}^{2}$, and so forth.

We can of course consider $\operatorname{Pr}_{V_{n}}$ as a measure on $\Omega_{\mathbf{Z}^{d}}$. Then for $\beta<\beta_{c}$, the critical inverse temperature, $\lim _{n \rightarrow \infty} \operatorname{Pr}_{V_{n}}$ exists, and is the unique equilibrium measure for the infinite volume system (for the given $\beta$ ). We denote thermal averages with respect to this measure simply by $\langle\cdot\rangle$. For now we restrict our attention to the case $\beta<\beta_{c}$. However, our method can be applied for pure states with finite susceptibility.

For each $n$ we choose a neighborhood $D_{n}$ of $B_{n}$ in which we shall delete the spins from the summation defining $M_{V_{n}}$. The object is to choose $D_{n}$ so small that this has a negligible effect on the distribution of $m_{V_{n}}$, but also thick enough that the remaining spins are not significantly affected by variation of the coupling on $B_{n}$.

Fix an increasing sequence of integers $\left\{L_{n} \mid n \in \mathbf{N}\right\}$ and define

$$
D_{n}=\left\{i \in V_{n}|| i-j \mid \leqq L_{n} \text { some } j \in B_{n}\right\} \text {. }
$$

We denote $M_{V_{n} \mid D_{n}}$ by $M_{V_{n}}^{\prime}, M_{V_{n-1} \mid D_{n}}$ by $M_{V_{n-1}}^{\prime \prime}$ and $M_{U_{n-1} \mid D_{n}}$ by $M_{U_{n-1}}^{\prime \prime}$. We let $m_{V_{n}}^{\prime}, m_{V_{n-1}}^{\prime \prime}$ and $m_{U_{n-1}}^{\prime \prime}$ denote the corresponding centered and normalised random variables. For each $n$, let $\mu_{n}$ denote the law of $m_{V_{n}}$ under $\operatorname{Pr}_{V_{n}}$, and let $v_{n}$ denote the law of $m_{U_{n}}$ under $\operatorname{Pr}_{U_{n}}$. Let $\lambda_{n}$ be given by

$$
\lambda_{n}=\sigma_{V_{n-1}}\left(M_{V_{n-1}}\right) /\left(\sigma_{V_{n-1}}^{2}\left(M_{V_{n-1}}\right)+\sigma_{U_{n-1}}^{2}\left(M_{U_{n-1}}\right)\right)^{1 / 2} \text {. }
$$

In order to display the insensitivity of the distribution of, say, $m_{V_{n}}^{\prime}$ to changes in the couplings or field at sites in $B_{n}$, we will use certain combinations of the correlation inequalities which we now recall. For an expository account of such correlation inequalities, see [GlJ or Sim].

We begin with the second Griffiths inequalities in a form due to Dunlop and Newman [DuNe] valid for one and two component systems. Let $\mathscr{F}$ denote the set 
of non-negative, non-decreasing functions $f$ on $[0, \infty)$. Let $\mathscr{G}_{1}^{N}$ denote the set of functions $F$ on $\mathbf{R}^{N}$ of the form

$$
F\left(s_{1}, \ldots, s_{N}\right)=\operatorname{sgn}\left(s_{1}\right)^{p_{1}} f_{1}\left(\left|s_{1}\right|\right) \ldots \operatorname{sgn}\left(s_{N}\right)^{p_{N}} f_{N}\left(\left|s_{N}\right|\right),
$$

where each $f_{j} \in \mathscr{F}$ and each $p_{j}$ is either 1 or 2 . Let $\mathscr{G}_{2}^{N}$ denote the set of functions $F$ on $\left(\mathbf{R}^{2}\right)^{N}$ of the form

$$
F\left(s_{1}, \ldots, s_{N}\right)=\cos \left(m_{1} \theta_{1}+\ldots+m_{N} \theta_{N}\right) f_{1}\left(r_{1}\right) \ldots f_{N}\left(r_{N}\right),
$$

where each $m_{j}$ is an integer, each $f_{j} \in \mathscr{F}$, and we have used polar coordinates on the right.

Then for all ferromagnetic spin systems with one or two components which are based on a reflection invariant reference measure $\mu$,

$$
\left\langle F\left(S_{i_{1}}, \ldots, S_{i_{N}}\right) G\left(S_{i_{1}}, \ldots, S_{i_{N}}\right)\right\rangle_{V} \geqq\left\langle F\left(S_{i_{1}}, \ldots, S_{i_{N}}\right)\right\rangle_{V}\left\langle G\left(S_{i_{1}}, \ldots, S_{i_{n}}\right)\right\rangle_{V}
$$

for all $\left\{i_{1}, \ldots, i_{N}\right\} \subset V \subset \mathbf{Z}^{d}$ and all $F, G \in \mathscr{G}_{D}^{N}, D=1,2$.

The most basic correlation function is the truncated two-point function

$$
\left\langle S_{i} ; S_{j}\right\rangle_{V}=\left\langle S_{i} \cdot S_{j}\right\rangle_{V}-\left\langle S_{i}\right\rangle_{V} \cdot\left\langle S_{j}\right\rangle_{V} .
$$

Certain inequalities which yield monotonicity of this and other correlations functions in the parameters - couplings and field strengths - of ferromagnetic systems are useful to us. For example, let $\left\langle S_{i} ; S_{j}\right\rangle_{V}(h)$ denote the truncated twopoint function considered as a function of the applied field $h$. In some cases

$$
\left\langle S_{i} ; S_{j}\right\rangle_{V}(h) \geqq\left\langle S_{i} ; S_{j}\right\rangle_{V}\left(h^{\prime}\right) \quad \forall h^{\prime}-h \geqq 0, h \geqq 0 .
$$

When $D=1$, this follows, for example, from the GHS inequality [GHS, ElNe] which states that for all ferromagnetic spin systems with scalar spins based on a reference measure $\mu$ of the form $d \mu(s)=e^{-\phi(s)} d s$ with $\phi$ even and continuously differentiable and with $\frac{d}{d s} \phi(s)$ convex on $(0, \infty)$, we have that

$$
\begin{aligned}
0 \leqq & \left\langle S_{i} S_{j} S_{k}\right\rangle_{V} \leqq\left\langle S_{i} S_{j}\right\rangle_{V}\left\langle S_{k}\right\rangle_{V}+\left\langle S_{i} S_{k}\right\rangle_{V}\left\langle S_{j}\right\rangle_{V} \\
& +\left\langle S_{j} S_{k}\right\rangle_{V}\left\langle S_{i}\right\rangle_{V}-2\left\langle S_{i}\right\rangle_{V}\left\langle S_{j}\right\rangle_{V}\left\langle S_{k}\right\rangle_{V}
\end{aligned}
$$

for all $i, j, k \in V \subset \mathbf{Z}^{d}$. Of course, we only need $\mu$ to be a weak limit of such measures. In case $D>1$, we say the GHS inequality holds in case for any fixed $u \in \mathbf{R}^{D}$, (3.11) holds with each $S_{m}$ replaced by $u \cdot S_{m}$, and the averages are conditional averages given the configuration of the components of the spins orthogonal to $u$, with respect to the orthogonality defined by the $J_{i, j}$. We shall have to assume that they are all multiples of one another to do this consistently. Then the parallel and orthogonal components of the spin decouple in the Hamiltonian, so by conditioning on the other components of the spins, it is easy to extend the above criteria for validity of the GHS inequality in the scalar case to the multicomponent case.

A number of other correlation inequalities are useful to us, in particular the Lebowitz inequality [Le74], and Bricmont's inequalities [Bri], as well as various Gaussian domination inequalities in [Ne75b, BFS] - which apply for multi component systems as well. These inequalities provide monotonicity of the truncated two-point function in the couplings, at zero field at least. Instead of recalling them now, we simply cite them when we actually use them. 
Finally, we will require some inequalities expressing the decay of correlations. In many instances, we only need the finite susceptibility condition; i.e. for some finite constant $C$

$$
\sum_{i \in V^{\prime}}\left\langle S_{i} ; S_{j}\right\rangle_{V} \leqq C
$$

for all $V^{\prime} \subset V$ and all $j \in V^{\prime}$.

In other cases we require more: For any $A \subset V \subset \mathbf{Z}^{d}$, let $\mathscr{B}_{A}$ be the $\sigma$-algebra generated by the spins in $A$; that is, $\mathscr{B}_{A}=\sigma\left\{S_{i} \mid i \in A\right\}$. Suppose that whenever $F$ is bounded and $\mathscr{B}_{A}$ measurable and $G$ is bounded and $\mathscr{B}_{B}$ measurable with $|A|$, $|B|<\infty$, there are finite positive constants $c(|A|,|B|)$ and $r(|A|,|B|)$, which are independent of $F, G$ and $V$, and depend on the couplings and applied fields only through $|J|$ and $|h|$, so that

$$
\left|\langle F G\rangle_{V}-\langle F\rangle_{V}\langle G\rangle_{V}\right| \leqq\|F\|_{\infty}\|G\|_{\infty} c(|A|,|B|) d(A, B)^{-r(|A|,|B|)} .
$$

Then we say our spin system has decay of correlations with rate $r(|A|,|B|)$ (at least). We only require this for $|A|,|B| \leqq 2$ below - at least when we are studying fluctuations in the magnetization. Therefore we never require bounds on the growth of $c(m, n)$ and $r(m, n)$ as we would in a perturbative approach.

Lemma 3.1. Consider a spin system with a bounded applied field. Suppose

$$
\left\langle S_{i} ; S_{j}\right\rangle_{V} \geqq 0 \quad \forall i, j \in V
$$

and that the finite susceptibility condition (3.12) holds. Then there exist constants $c_{1}$ and $c_{2}$ with $0<c_{1} \leqq c_{2}<\infty$ such that

$$
c_{1}\left|V^{\prime}\right| \leqq \sigma_{V}^{2}\left(M_{V^{\prime}}\right) \leqq c_{2}\left|V^{\prime}\right| \quad \forall V^{\prime} \subset V \subset \mathbf{Z}^{d} .
$$

Proof. Note that

$$
\sigma_{V}^{2}\left(M_{V^{\prime}}\right)=\sum_{i, j \in V^{\prime}}\left\langle S_{i} ; S_{j}\right\rangle_{V}
$$

The upper bound follows immediately from (3.12) with $c_{2}=C$.

For the lower bound, note that by (3.14),

$$
\sigma_{V}^{2}\left(M_{V^{\prime}}\right) \geqq \sum_{i \in V^{\prime}}\left\langle S_{i} ; S_{i}\right\rangle_{V}
$$

For each $i \in V_{n}$, let $\mathscr{B}_{\partial i}$ denote the $\sigma$-field generated by the spins neighboring $S_{i}$ :

$$
\mathscr{B}_{\partial i}=\sigma\left\{S_{j}|j \in V,| i-j \mid=1\right\} \text {. }
$$

Clearly the conditional expectation $\left\langle\left|S_{i}-\left\langle S_{i}\right\rangle_{V_{n} ; \lambda}\right|^{2} \mid \mathscr{B}_{\partial i}\right\rangle_{V_{m} ; \lambda}$ is bounded below, as a function of the neighboring configuration, by a positive constant depending only on $\beta,|J|,|h|, r(\mathscr{S})$ and $\sup \left\{a^{2} \mu(|S| \geqq a) \mid a>0\right\}$. The last quantity is positive by our assumptions on $\mu$. Denote this constant by $c_{1}$, and then by (3.16), the lower bound in (3.15) follows.

Lemma 3.2. Consider a spin system for which either (3.10), monotonicity in the applied field, is satisfied, or for which decay of correlations, (3.13), holds with $r(2,1)$ $>3 D$. Let $|h|$ be given, and for any specification of $h_{i}$ for $i \in V$ with $\left|h_{i}\right| \leqq|h|$ for all $i \in V$, define $X_{h}=\sum_{i \in V} h_{i} \cdot S_{i}$. Then for some finite constant $C$ independent of $V$ and depending on the $h_{i}$ only through $|h|$,

$$
\left|\ln \left\langle e^{X_{h}}\right\rangle_{V}\right| \leqq C .
$$


Remark. Under the monotonicity condition, this result is due to Newman [Ne75a]. Proof. Since the region is finite, $\ln \left\langle e^{h m_{V}}\right\rangle_{V}$ is a smooth function of $h$. By Taylor's theorem,

$$
\ln \left\langle e^{h m_{V}}\right\rangle_{V}=\frac{h^{2}}{2}\left\langle e^{\xi m_{V}}\right\rangle_{V}^{-2}\left(\left\langle m_{V}^{2} e^{\xi m_{V}}\right\rangle_{V}\left\langle e^{\xi m_{V}}\right\rangle_{V}-\left\langle m_{V} e^{\xi m_{V}}\right\rangle_{V}^{2}\right)
$$

for some $\xi$ with $0 \leqq \xi \leqq h$. The term multiplying $\frac{h^{2}}{2}$ is precisely the ratio of the variance of $M_{V}$ in an applied field with $h_{i}=\left(\xi / \sigma_{V}\right) u$ for all $i \in V$ to the variance of $M_{V}$ in zero applied field.

Clearly when (3.10) holds, this is non-negative and no more than unity, so the result is established.

Now suppose that (3.13) holds with $r(2,1)>3 D$. Clearly to establish (3.17), it suffices to establish that the variance of the magnetization in an applied field is continuous in the applied field strength. This can be established by a method of Lebowitz [Le72]:

By considering correlations for different combinations of components separately, it suffices to treat scalar spins. The derivative of $\left\langle S_{i} ; S_{j}\right\rangle$ in the strength of the applied field at site $k$ is

$$
\left\langle\left(S_{i}-\left\langle S_{i}\right\rangle_{V}\right)\left(S_{j}-\left\langle S_{j}\right\rangle_{V}\right) S_{k}\right\rangle_{V}-\left\langle S_{i} ; S_{j}\right\rangle_{V}\left\langle S_{k}\right\rangle_{V}
$$

which, upon expansion, is seen to be symmetric in $i, j$ and $k$. Since it is also clearly bounded in absolute value by $(r(\mathscr{S}))^{3} c(2,1) d((i, j),(k))^{-r(2,1)}$, we may permute $i, j$ and $k$ and then take the best case. Let $d_{1} \leqq d_{2} \leqq d_{3}$ denote the lengths of the sides of the triangle with vertices $i, j$ and $k$. The best case is when the distance is $d_{2}$. Since $d_{2}^{-1} \leqq(4 / 3)\left(d_{1} d_{2} d_{3}\right)^{-1 / 3}$, we obtain a bound of the form

$$
(r(\mathscr{S}))^{3} c(2,1)\left(d_{1} d_{2} d_{3}\right)^{-r(2,1) / 3} \text {. }
$$

Thus for some constant $K$ depending on the applied field only through its strength,

$$
\sum_{i, j, k \in V}\left|\left\langle\left(S_{i}-\left\langle S_{i}\right\rangle_{V}\right)\left(S_{j}-\left\langle S_{j}\right\rangle_{V}\right) S_{k}\right\rangle_{V}-\left\langle S_{i} ; S_{j}\right\rangle_{V}\left\langle S_{k}\right\rangle_{V}\right| \leqq K|V| .
$$

Lemma 3.3. Consider a spin system for which (3.17) and (3.14) are satisfied. Let $\left\{A_{n} \mid n \in \mathbf{N}\right\},\left\{A_{n}^{\prime} \mid n \in \mathbf{N}\right\}$ and $\left\{F_{n} \mid n \in \mathbf{N}\right\}$ be any sequences of regions with $A_{n} \cup A_{n}^{\prime} \subset F_{n}$ $C \mathbf{Z}^{d} \forall n \in \mathbf{N}, \lim \left|A_{n}\right|=\infty$, and $\lim _{n \rightarrow \infty}\left|A_{n} \Delta A_{n}^{\prime}\right| /\left|A_{n}\right|=0$. Let $X_{n}$ and $Y_{n}$ denote $\left(M_{A_{n}}\right.$ $\left.-\left\langle M_{A_{n}}\right\rangle_{F_{n}}\right) / \sigma_{F_{n}}\left(M_{A_{n}}\right)$ and $\left(M_{A_{n}^{\prime}}-\left\langle M_{A_{n}^{\prime}}\right\rangle_{F_{n}}\right) / \sigma_{F_{n}}\left(M_{A_{n}^{\prime}}\right)$ respectively. Then

for all $\in[0,1]$.

$$
\lim _{n \rightarrow \infty}\left|\ln \left\langle e^{h X_{n}}\right\rangle_{F_{n}}-\ln \left\langle e^{h Y_{n}}\right\rangle_{F_{n}}\right|=0
$$

Proof. We define $W_{n}$ by $W_{n}=X_{n}-Y_{n}$. Clearly

$$
\begin{aligned}
W_{n}= & \sum_{i \in A_{n} \backslash A_{n}^{\prime}}\left(S_{i}-\left\langle S_{i}\right\rangle_{F_{n}}\right) / \sigma_{F_{n}}\left(M_{A_{n}}\right)-\sum_{i \in A_{n}^{\prime} \backslash A_{n}}\left(S_{i}-\left\langle S_{i}\right\rangle_{F_{n}}\right) / \sigma_{F_{n}}\left(M_{A_{n}}\right) \\
& +\left(\sigma_{F_{n}}\left(M_{A_{n}}\right) / \sigma_{F_{n}}\left(M_{A_{N}^{\prime}}\right)-1\right) Y_{n}=W_{n}^{(1)}+W_{n}^{(2)}+W_{n}^{(3)} .
\end{aligned}
$$

From Lemma 3.1 we have $\sigma_{F_{n}}^{2}\left(W_{n}^{(1)}\right)+\sigma_{F_{n}}^{2}\left(W_{n}^{(2)}\right) \leqq c_{2}\left|A_{n} \Delta A_{n}^{\prime}\right|$ and clearly $\sigma_{F_{n}}^{2}\left(W_{n}^{(3)}\right)$ $\leqq\left(\sigma_{F_{n}}\left(M_{A_{n}}\right) / \sigma_{F_{n}}\left(M_{A_{N}^{\prime}}\right)-1\right)^{2}$. Again using Lemma 3.1 and obvious estimates, we 
obtain $\sigma_{F_{n}}^{2}\left(W_{n}\right) \leqq K \frac{\left|A_{n} \Delta A_{n}^{\prime}\right|}{\left|A_{n}\right|}$ where, as in the rest of the proof, $K$ denotes a finite constant, changing from line to line, depending only on $c_{1}$ and $c_{2}$.

Next, for $\xi \in[0,1]$ and $h \geqq 0$, define $f_{n}(\xi, h)=\left\langle e^{h\left(X_{n}+\xi W_{n}\right)}\right\rangle_{F_{n}}$. It suffices to prove that $\lim _{n \rightarrow \infty}\left(f_{n}(1, h)-f_{n}(0, h)\right)=0$, and so it suffices to prove that $\lim _{n \rightarrow \infty}\left|\frac{\partial}{\partial \xi} f_{n}(\xi, h)\right|=0$ uniformly in $\xi$.

By (3.17), $f(\xi, h) \leqq C$ uniformly in $0 \leqq \xi, h \leqq 2$. Thus

$$
\begin{aligned}
\left|\frac{\partial}{\partial \xi} f_{n}(\xi, h)\right| & =h\left|\left\langle W_{n} e^{h\left(X_{n}+\xi W_{n}\right)}\right\rangle_{F_{n}}\right| \leqq\left\langle W_{n}^{2}\right\rangle_{F_{n}}^{1 / 2}\left(f_{n}(\xi, 2 h)\right)^{1 / 2} \\
& \leqq h C K\left(\frac{\left|A_{n} \Delta A_{n}^{\prime}\right|}{\left|A_{n}\right|}\right)^{1 / 2}
\end{aligned}
$$

by the Schwarz inequality and the estimates above since $W_{n}$ has zero mean.

Lemma 3.4. Consider a spin system satisfying either of the following sets of conditions: (I) There is zero applied field, the second Griffiths inequality holds, the GHS inequality holds (in the sense specified following (3.10) for multicomponent systems) and the infinite volume state has finite susceptibility.

(II) Decay of correlations (3.13) holds with $r(2,1)>3 D$ and $\left\langle S_{i} ; S_{j}\right\rangle \geqq 0$ for all $i$ and $j$.

Then

$$
\begin{aligned}
& \lim _{n \rightarrow \infty}\left|\ln \left\langle e^{h m_{V_{n}}^{\prime}}\right\rangle_{V_{n}}-\ln \left\langle e^{h m_{V_{n}}^{\prime}}\right\rangle_{V_{n} ; 0}\right|=0, \\
& \lim _{n \rightarrow \infty}\left|\ln \left\langle e^{h m_{V_{n}}^{\prime \prime}}\right\rangle_{V_{n}}-\ln \left\langle e^{h m_{V_{n}}^{\prime \prime}}\right\rangle_{V_{n} \backslash B_{n}}\right|=0
\end{aligned}
$$

for all $h \in[0,1]$. Moreover, the analogs of (3.19) and (3.20) for $U_{n}$ also holds.

Proof. We first treat both sets of conditions in the special case $D=1$ to avoid an excess of indices. We then separately deal with the complications entailed by $D>1$. To establish (3.19), define $f_{n}(\lambda, h)=\left\langle e^{h m_{V_{n}}^{\prime}}\right\rangle_{V_{n} ; \lambda}$. Then

$$
\begin{aligned}
\frac{\partial}{\partial \lambda} f_{n}(\lambda, h)= & \beta \sum_{k, k^{\prime} \in B_{n}}\left\langle e^{h m_{V}^{\prime}}\right\rangle_{V_{n} ; \lambda} \\
& \times\left[\frac{\left\langle J_{k, k^{\prime}}\left(S_{k}, S_{k^{\prime}}\right) e^{\left.h m_{V_{n}}^{\prime}\right\rangle_{V_{n} ; \lambda}}\right.}{\left\langle e^{h m_{V_{n}}^{\prime}}\right\rangle_{V_{n} ; \lambda}}-\left\langle J_{k, k^{\prime}}\left(S_{k}, S_{k^{\prime}}\right)\right\rangle_{V_{n} ; \lambda}\right] .
\end{aligned}
$$

The quantity in braces is the difference between the thermal averages of $J_{k, k^{\prime}}\left(S_{k}, S_{k^{\prime}}\right)$ under the law $\operatorname{Pr}_{V_{n} ; \lambda}$ as it is, and the law $\operatorname{Pr}_{V_{n} ; \lambda}$ perturbed by a magnetic field of strength $h / \sigma_{V_{n}}\left(M_{V_{n}}^{\prime}\right)$ on $V_{n} \backslash D_{n}$. Since this location is well separated from $k$ and $k^{\prime}$, which are in $B_{n}$, the difference is small. To estimate it, we differentiate in $h$ : Define $g_{n, k, k^{\prime}}(h, \lambda)$ by

Then

$$
g_{n, k, k^{\prime}}(h, \lambda)=\frac{\left\langle J_{k, k^{\prime}}\left(S_{k}, S_{k^{\prime}}\right) e^{h m_{V_{n}}}\right\rangle_{V_{n} ; \lambda}}{\left\langle e^{h m_{V_{n}}}\right\rangle_{V_{n} ; \lambda}}
$$

$$
\begin{aligned}
\frac{\partial}{\partial h} g_{n, k, k^{\prime}}(h, \lambda)= & \frac{\beta}{\sigma_{V_{n}}\left(M_{V_{n}}^{\prime}\right)} \sum_{j \in V_{n} \backslash D_{n}}\left\langle e^{\left.h m_{V_{n}}^{\prime}\right\rangle_{V_{n} ; \lambda}^{-2}}\right. \\
& -\left\langle J_{k, k^{\prime}}\left(S_{k}, S_{k^{\prime}}\right) e^{\left.\left.h m_{V_{n}}^{\prime}\right\rangle_{V_{n} ; \lambda}\left(S_{j} e^{h m_{V_{n}}^{\prime}}\right\rangle_{V_{n} ; \lambda}\right]}\right.
\end{aligned}
$$


Now consider the first set of conditions. By the second Griffiths inequality, $\frac{\partial}{\partial h} g_{n, k, k^{\prime}} \geqq 0$. By the GHS inequality

$$
\begin{aligned}
& \left\langle e^{h m_{V_{n}}^{\prime}}\right\rangle_{V_{n} ; \lambda}^{-2}\left[\left\langle S_{k} S_{k^{\prime}} S_{j} e^{h m_{V_{n}}^{\prime}}\right\rangle_{V_{n} ; \lambda}\left\langle e^{h m_{V_{n}}^{\prime}}\right\rangle_{V_{n} ; \lambda}-\left\langle S_{k} S_{k^{\prime}} e^{h m_{V_{n}}^{\prime}}\right\rangle_{V_{n} ; \lambda}\left\langle S_{j} e^{\left.h m_{V_{n}}^{\prime}\right\rangle_{V_{n} ; \lambda}}\right]\right. \\
& \leqq\left\langle e^{h m_{V_{n}}^{\prime}}\right\rangle_{V_{n} ; \lambda}^{-2}\left[\left\langle S_{k} ; S_{j} e^{h m_{V_{n}}^{\prime}}\right\rangle_{V_{n} ; \lambda}\left\langle S_{k^{\prime}} e^{h m_{V_{n}}^{\prime}}\right\rangle_{V_{n} ; \lambda}+\left\langle S_{k^{\prime}} ; S_{j} e^{h m_{V_{n}}}\right\rangle_{V_{n} ; \lambda}\left\langle S_{k} e^{h m_{V_{n}}^{\prime}}\right\rangle_{V_{n} ; \lambda}\right] \\
& \leqq\left\langle S_{k} ; S_{j}\right\rangle_{V_{n}}\left|\frac{\left\langle S_{k^{\prime}} e^{h m_{V_{n}}}\right\rangle_{V_{n} ; \lambda}}{\left\langle e^{h m_{V_{n}}}\right\rangle_{V_{n} ; \lambda}}\right|+\left\langle S_{k^{\prime}} ; S_{j}\right\rangle_{V_{n}}\left|\frac{\left\langle S_{k} e^{h m_{V_{n}}}\right\rangle_{V_{n} ; \lambda}}{\left\langle e^{h m_{V_{n}}}\right\rangle_{V_{n} ; \lambda}}\right| \text {. }
\end{aligned}
$$

The last line follows because

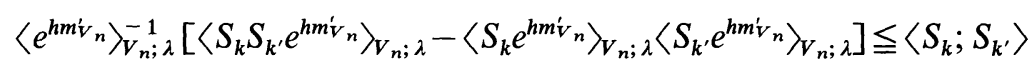

which in turn follows from the GHS inequality (monotonicity in the applied field) and the Lebowitz inequality at zero field (monotonicity in the coupling constant). Recall that at zero field, the latter inequality is a consequence of the former.

Next note that

$$
\left|\frac{\left\langle S_{k} e^{h m_{V_{n}}^{\prime}}\right\rangle_{V_{n} ; \lambda}}{\left\langle e^{h m_{V_{n}}}\right\rangle_{V_{n} ; \lambda}}\right| \leqq \frac{K h}{\sigma_{V_{n}}\left(M_{V_{n}}^{\prime}\right)}
$$

by the now familiar differentiation and integration argument. Altogether then (with $K$ changing)

$$
0 \leqq \frac{\partial}{\partial h} g_{n, k, k^{\prime}}(h, \lambda) \leqq \frac{K h}{\left|V_{n} \backslash D_{n}\right|} \sum_{j \in V_{n} \backslash D_{n}}\left[\left\langle S_{k} ; S_{j}\right\rangle+\left\langle S_{k^{\prime}} ; S_{j}\right\rangle\right] .
$$

Clearly then $\sum_{k, k^{\prime} \in B_{n}} \frac{\partial}{\partial h} g_{n, k, k^{\prime}} \delta_{\left|k-k^{\prime}\right|, 1}$ tends to zero with $n$ as long as $L_{n}$ increases to infinity. This proves the lemma for $D=1$ under the first set of conditions.

Under the second set of conditons, note that by (3.13), we have that

$$
\left|\frac{\partial}{\partial h} g_{n, k, k^{\prime}}(h, \lambda)\right| \leqq \frac{\beta\left|V_{n}\right|}{\left(c_{1}\left|V_{n} \backslash D_{n}\right|\right)^{1 / 2}}|J|(r(\mathscr{S}))^{3} c(2,1) L_{n}^{-r(2,1)} .
$$

Two applications of the mean value theorem yield (3.19) as long as we take $L_{n}$ to be the integral part of $\left|V_{n}\right|^{p / D}$ for $p$ sufficiently close to 1 .

Now consider the case $D>1$ under the first set of conditions; under the second set, nothing more need be said. We reduce to the case $D=1$ simply by conditioning on the components of the spins orthogonal to $h$. Uniform estimates on these conditional moment generating functions clearly yield the desired estimates by averaging.

Next note that since $\left\langle e^{h m_{V_{n}}^{\prime \prime}}\right\rangle_{V_{n} \mid B_{n}}$ is obtained from $\left\langle e^{h m_{V_{n}}^{\prime \prime}}\right\rangle_{V_{n}}$ by "turning off" couplings, an entirely similar argument also proves (3.20). Clearly the results for $U_{n}$ are established by interchanging $V_{n}$ and $U_{n}$.

Lemma 3.5. Under the same hypotheses as Lemma 3.4,

$$
\lim _{n \rightarrow \infty}\left|\left\langle M_{V_{n}}^{\prime}\right\rangle_{V_{n}}-\left\langle M_{V_{n-1}}^{\prime \prime}\right\rangle_{V_{n-1}}-\left\langle M_{U_{n-1}}^{\prime \prime}\right\rangle_{U_{n-1}}\right| / \sigma_{V_{n}}\left(M_{V_{n}}^{\prime}\right)=0 .
$$

Proof. This is essentially a corollary of the proof of Lemma 3.4. Actually, under the first set of conditions, all the means vanish, and there is nothing to prove. As for the second set of conditions, note that the usual differenitation argument leads to essentially the quantity on the right side of (3.22), except that now the factor of $1 / \sigma_{V_{n}}\left(M_{V_{n}}^{\prime}\right)$ comes from (3.23) itself, and not from a small field size. 
Lemma 3.6. Suppose that either the first set of hypotheses for Lemma 3.4 are satisfied, or that (3.13) holds with $r(2,1), r(2,2)>2 D-1$, or that

Then

$$
\lim _{n \rightarrow \infty} \sigma_{V_{n}} /\left|V_{n}\right|=\lim _{n \rightarrow \infty} \sigma_{U_{n}} /\left|U_{n}\right|=a>0 .
$$

$$
\lim _{n \rightarrow \infty}\left|\sigma_{V_{n}}^{2}\left(M_{V_{n}}^{\prime}\right)-\sigma_{V_{n-1}}^{2}\left(M_{V_{n-1}}^{\prime \prime}\right)-\sigma_{U_{n-1}}^{2}\left(M_{U_{n-1}}^{\prime \prime}\right)\right| / \sigma_{V_{n}}^{2}\left(M_{V_{n}}^{\prime}\right)=0 .
$$

Proof. Note that

$$
\begin{aligned}
\frac{d}{d \lambda} & \sum_{i, j \in V_{n}^{\prime}}\left\langle S_{i} ; S_{j}\right\rangle_{V_{n} ; \lambda} \\
= & \sum_{i, j \in V_{n}^{\prime} k, k^{\prime} \in B_{n},\left|k-k^{\prime}\right|=1}\left\{\left\langle S_{i} \cdot S_{j} J_{k, k^{\prime}}\left(S_{k}, S_{k^{\prime}}\right)\right\rangle_{V_{n}}-\left\langle S_{i} \cdot S_{j}\right\rangle_{V_{n}}\left\langle J_{k, k^{\prime}}\left(S_{k}, S_{k^{\prime}}\right)\right\rangle_{V_{n}}\right. \\
& -\left(\left\langle S_{i} J_{k, k^{\prime}}\left(S_{k}, S_{k^{\prime}}\right)\right\rangle_{V_{n}}-\left\langle S_{i}\right\rangle_{V_{n}}\left\langle J_{k, k^{\prime}}\left(S_{k}, S_{k^{\prime}}\right)\right\rangle_{V_{n}}\right) \cdot\left\langle S_{j}\right\rangle_{V_{n}} \\
& \left.-\left(\left\langle S_{j} J_{k, k^{\prime}}\left(S_{k}, S_{k^{\prime}}\right)\right\rangle_{V_{n}}-\left\langle S_{j}\right\rangle_{V_{n}}\left\langle J_{k, k^{\prime}}\left(S_{k}, S_{k^{\prime}}\right)\right\rangle_{V_{n}}\right) \cdot\left\langle S_{i}\right\rangle_{V_{n}}\right\}
\end{aligned}
$$

In case the first condition holds, the Lebowitz inequality enables us to bound each term in this sum by a multiple of

$$
\left\langle S_{i} ; S_{k}\right\rangle\left\langle S_{j} ; S_{k^{\prime}}\right\rangle+\left\langle S_{j} ; S_{k}\right\rangle\left\langle S_{i} ; S_{k^{\prime}}\right\rangle \text {. }
$$

Now finite susceptibility and our lower bounds for $\sigma_{V}^{2}\left(M_{V_{n}}^{\prime}\right)$ yield the first assertion. The remaining assertions are readily verified.

Essentially by combining our lemmas, we have the theorem below. Note that for $D=1$, we do not require $\lim _{n \rightarrow \infty} \sigma_{V_{n}} /\left|V_{n}\right|$ to exist. It is easy to arrange the couplings so that this limit will not exist.

Theorem 3.1. Consider a spin system satisfying the hypotheses of Lemma 3.4. In case $D=1$, suppose that the hypotheses of Lemma 3.6 is satisfied. In case D>1, suppose that

$$
\lim _{n \rightarrow \infty}\left[\sigma_{V_{n}}\left(M_{V_{n}}\right)\right]^{2} /\left|V_{n}\right|=\lim _{n \rightarrow \infty}\left[\sigma_{U_{n}}\left(M_{U_{n}}\right)\right]^{2} /\left|U_{n}\right|=A
$$

exists and is nondegenerate. Let $\mathscr{Z}_{n}=\left\{m_{V_{n, j}} \mid j \in \mathbf{N}\right\}$ under the infinite volume law. Then

in distribution.

$$
\lim _{n \rightarrow \infty} \mathscr{Z}_{n}=\mathscr{G}
$$

Proof. Note first that the hypotheses of Lemma 3.4 imply the hypotheses of Lemmas 3.1, 3.2, 3.3 and 3.5. By Lemmas 3.1 through 3.6, or 3.1 through 3.5 and (3.25), all of the hypotheses of Theorem 2.5 are satisfied provided we take the integers $L_{n}$ defining the regions $D_{n}$ to be the integral part of $\left|V_{n}\right|^{p / D}$ for some appropriate power $p$ with $0<p<1$.

To see that the condition (2.31) is satisfied, consider explicitly the case $D=1$, and note that with $\lambda_{n, 1}$ given by (3.7), and $\mu_{n, j}$ denoting the law of $m_{V_{n, j}}$,

$$
\begin{aligned}
& \left|\Lambda_{\mu_{n, 1}}(h)-\Lambda_{\mu_{n-1,1} *_{n} \mu_{n-1,2}}(h)\right| \\
& \leqq\left|\ln \left\langle e^{h m_{V_{n}}}\right\rangle-\ln \left\langle e^{h m_{V_{n}}^{\prime}}\right\rangle\right|+\left|\ln \left\langle e^{h m_{V_{n}}^{\prime}}\right\rangle-\ln \left\langle e^{h m_{V_{n}}^{\prime}}\right\rangle_{V_{n} ; 0}\right| \\
& +\left|\ln \left\langle e^{h m_{V n}^{\prime}}\right\rangle_{V_{n} ; 0}-\ln \left\langle e^{h \lambda_{n, 1} m_{V_{n-1}}^{\prime \prime}}\right\rangle_{V_{n-1}}-\ln \left\langle e^{h\left(1-\lambda_{n, 1}^{2}\right)^{1 / 2} m_{U_{n-1}}^{\prime \prime}}\right\rangle_{U_{n-1}}\right| \\
& +\left|\ln \left\langle e^{h \lambda_{n, 1} m_{V_{n-1}}}\right\rangle_{V_{n-1}}-\ln \left\langle e^{h\left(1-\lambda_{n, 1}^{2}\right)^{1 / 2} m_{U_{n-1}^{\prime}}^{\prime}}\right\rangle_{U_{n-1}}-\Lambda_{\mu_{n-1,1} *_{n, 1} \mu_{n-1,2}}(h)\right| \text {. }
\end{aligned}
$$


The terms following the inequality sign tend to zero by, respectively, Lemma 3.3, Lemma 3.4, Lemma 3.5 together with Lemma 3.6, and Lemma 3.3 again.

That condition (2.32) holds follows in the same way - in fact neighboring blocks are the hardest case.

We now briefly discuss the application of our method to FKG systems. For background on these, see [ $\mathrm{Ne80}$ ] and [Ne83]. We will continue to use the notations established above, but we will no longer assume that the law is given in terms of a Hamiltonian as above. We shall only assume that the spins are FKG variables.

Newman has proved $[\mathrm{Ne} 80]$ that for finite variance $\mathrm{FKG}$ variables $Y_{1}, \ldots, Y_{N}$,

$$
\left|\left\langle\exp \left(i \sum_{k=1}^{N} r_{k} Y_{k}\right)\right\rangle-\Pi\left\langle\exp \left(i r_{k} Y_{k}\right)\right\rangle\right| \leqq \frac{1}{2} \sum_{k=1}^{N} \sum_{m=1, m \neq k}^{N}\left|r_{k}\right|\left|r_{m}\right| \operatorname{cov}\left(Y_{k}, Y_{m}\right) \text {. }
$$

He has used this to prove a central limit theorem for translation invariant FKG systems under a finite susceptibility condition. To establish a Gaussian limit in our approach, we only need (3.27) for $N=2$ to verify the condition (2.23) of Theorem 2.4. The conditions (2.6) and (2.7) of Theorem 2.1 are readily checked in FKG systems with finite susceptibility - note that the function $\varphi_{L}$ in (2.6) is monotone. The condition (2.8) follows from using the two point domination arguments [Sim, Le73] on which Newman's inequality (3.27) is based, to control the fourth moment in (2.8) in terms of the two-point function. This argument is easy, but it requires somewhat more than finite susceptibility; the two point function must decay with the distance at an inverse power exceeding twice the dimension of the underlying lattice. While Theorem 2.1 can be refined so that less than condition (2.8) is required, this is probably already enough to allow us to treat interesting random field problems.

Acknowledgements. We would like to thank Gian-Fausto Dell'Antonio, Persi Diaconis, Elliott Lieb, Michael Loss, David Ruelle, Thomas Spencer, Dan Stroock, and Jan Wehr for interesting discussions on these and related subjects. Some work related to this paper was done over the Summer of 1989. E.C. would like to thank the Università degli studi di Roma "La Sapienza," and A.S. would like to thank I.H.E.S. for hospitality and the chance to discuss this work in its early stages.

\section{References}

[BaEm] Bakry, D., Emery, M.: Diffusions hypercontractives. Seminaire de Probabilities XIX, Lect. Notes in Math. vol. 1123, pp. 179-206. Berlin, Heidelberg, New York: Springer 1985

[Bar] Barron, A.R.: Entropy and the central limit theorem. Ann. Prob. 14, 336-342 (1986)

[Bla] Blachman, N.M.: The convolution inequality for entropy powers. IEEE Trans. Info. Thy. 2, 267-271 (1965)

[Bri] Bricmont, J.: Correlation inequalities for two component fields. Ann. Soc. Sr. Bru., 90, 245-252 (1976)

[Bro] Brown, L.D.: A proof of the central limit theorem motivated by the Cramer-Rao inequality. In: Statistics and Probability: Essays in Honor of C.R. Rao. Kallianpur et al. (eds.) pp. 314-328 Amsterdam: North-Holland 1982

[BFS] Brydges, D., Fröhlich, J., Spencer, T.: A random walk representation of classical spin systems and correlation inequalities. Commun. Math. Phys. 83, 123-150 (1982)

$[\mathrm{BrKu}]$ Bricmont, J., Kupiainen, A.: Phase transition in the $3 d$ random field Ising model. Commun. Math. Phys. 116, 539-572 (1988) 
[Car] Carlen, E.A.: Superadditivity of Fisher's Information and Logarithmic Sobolev Inequalities. J. Funct. Anal. (to appear)

[CaLo] Carlen, E.A., Loss, M.: Extremals of functionals with competing symmetries. J. Funct. Anal. 88, 437-456 (1990)

[CaSo] Carlen, E.A., Soffer, A.: Entropy production by convolution and central limit theorems with strong rate information, Princeton preprint, in preparation

[Cra] Cramér, H.: Über eine Eigenschaft der normalen Verteilungsfunktion. Math. Zeit. 41, 405-414 (1936)

[Csi] Csiszar, I.: Informationstheoretische Konvergenzbegriffe im Raum der Wahrscheinlichkeitsverteilungen, Publications of the Mathematical Institute, Hungarian Academy of Sciences, VII, Series A, 137-157 (1962)

[Dem] Dembo, A.: Information inequalities and uncertainty principles, Stanford University Technical Report No. 75, (1990)

[DeC] DeConinck, J.: On limit theorems for the bivariate (magnetization, energy) variable at the critical point. Commun. Math. Phys. 109, 53-59 (1984)

[Des] Desvillettes, L.: Entropy dissipation rate and convergence to equilibrium in Kinetic Equations. Commun. Math. Phys. 123, 687-702 (1989)

[DeSt] Deuschel, J.D., Stroock, D.W.: Large deviations. Boston: Academic Press 1989

[DN] Dunlop, F., Newman, C.: Multicomponent field theories and classical rotators. Commun. Math. Phys. 44, 223-235 (1975)

[DoVa] Donsker, M.D., Varadhan, S.R.S.: Asymptotic evaluation of certain Markov process expectations for large time, I. Comm. Pure Appl. Math. 28, 1-47 (1975)

[EINe] Ellis, R., Newman, C.: Necessary and sufficient conditions for the application of the GHS inequality with applications to analysis and probability. Trans. Am. Math. Soc. 237, 83-99 (1978)

[Ens] Enss, V.: Asymptotic completeness for quantum mechanical scattering I: Short range potentials. Commun. Math. Phys. 61, 285-291 (1978)

[Fis] Fisher, R.A.: Theory of statistical estimation. Proc. Cam. Phil. Soc. 22, 700-725 (1925)

[FKG] Fortuin, C.M., Kasteleyn, P.W., Ginibre, J.: Correlation inequalities on some partially ordered sets. Commun. Math. Phys. 22, 89-103 (1971)

[GaJL] Gallavotti, G., Jona-Lasinio, G.: Limit theorems for multidimensional Markov processes. Commun. Math. Phys. 41, 301-307 (1975)

[GaML] Gallavotti, G., Martin-Löf, A.: Block spin distribution for short range attractive Ising models. Nuovo Cim. 25 B, 425-441 (1975)

[Gaw] Gawedzki, K.: Block Spin Renormalization. In: Mathematics and Physics: Recent Results. Vol. I. L. Striet (ed.). Singapore: World Scientific 1983

[G1Ja] Glimm, J., Jaffe, A.: Quantum physics a functional integral point of view. New York: Springer 1981

[Gro] Gross, L.: Logarithmic Sobolev inequalities. Am. J. Math. 97, 1061-1083 (1975)

[Gri] Griffiths, R.B., Hurst, C.A., Sherman, S.: Concavity of magnetization of an Ising ferromagnet in a positive externfal field. J. Math. Phys. 11, 790-795 (1970)

[GPV] Guo, M.Z., Papanicolau, G.C., Varadhan, S.R.S.: Nonlinear diffusion limit for a system with nearest neighbor interactions. Commun. Math. Phys. 118, 31-67 (1988)

[HeNa] Hegerfeldt, G., Nappi, C.: Mixing properties of lattice systems. Commun. Math. Phys. 53, 1-7 (1977)

[Hol] Holley, R.: Free energy in a Markovian model of a lattice spin system. Commun. Math. Phys. 23, 8-28 (1971)

[JoL] Jona-Lasinio, J.: The renormalization group: A probabilistic view. Nuovo Cim. 26 B, 99-119 (1975)

[Kac] Kac, M.: On a characterization of the normal distribution. Am. J. Math. 61, 473-476 (1939)

[KeSh] Kelly, D.G., Sherman, S.: General Griffiths inequalities on correlations in Ising ferromagnetis. J. Math. Phys. 9, 466-484 (1968)

[Kul] Kullback, S.: A lower bound for discrimination information in terms of variation. IEEE Trans. Info. Thy. 4, 126-127 (1967)

[Le74] Lebowitz, J.: GHS and other Inequalities. Commun. Math. Phys. 35, 87-92 (1974)

[Le72] Lebowitz, J.: Bounds on the Correlations and Analyticity Properties of Ferromagnetic Ising Spin Systems. Commun. Math. Phys. 28, 313-321 (1972) 
[LePe] Lebowitz, J., Penrose, O.: Analytic and clustering properties of thermodynamic functions for classical lattice and continuum systems. Commun. Math. Phys. 11, 99-124 (1968)

[Lie78] Lieb, E.H.: Proof of an entropy conjecture of Wehrl. Commun. Math. Phys. 62, 35-41 (1978)

[Lie89] Lieb, E.H.: Gaussian kernels have Gaussian maximisers, Princeton preprint, 1989

[Lin59] Linnik, Ju.V.: An information theoretic proof of the central limit theorem with Lindeberg conditions. Theory Probab. Appl. 4, 288-299 (1959)

[Lin60] Linnik, Ju.V.: On certain connections of the information theory of C. Shannon and R. Fisher with the theory of symmetrization of random vectors, Transactions of the Second Prague Conference on Information Theory, 313-327, Publ. House Szech. Akad. Sci., Prague; New York: Academic Press 1960

[McK] McKean, H.: Speed of approach to equilibrium for Kac's caricature of a Maxwellian gas. Arch. Rat. Mech. Anal. 21, 342-367 (1966)

[Mou] Mourre, E.: Absence of singular spectrum of certain self-adjoint operators. Commun. Math. Phys. 78, 391-408 (1981)

[Ne75a] Newman, C.M.: Moment inequalities for ferromagnetic Gibbs distributions. J. Math. Phys. 16, 1956-1959 (1975)

[Ne80] Newman, C.M.: Normal Fluctuations and the FKG Inequalities. Commun. Math. Phys. 74, 129-140 (1980)

[Ne83] Newman, C.M.: A general central limit theorem for FKG systems. Commun. Math. Phys. 91, 75-80 (1983)

[Ne75b] Newman, C.M.: Gaussian correlation inequalities for ferromagnets. Z. Wahrsch. Gebiete 33, 75-93 (1975)

[Sap] Sapagov, N.A.: On independent terms of a sum of random variables which is distributed almost normally. Vestnik Leningrad Univ. 14, 78-105 (1959)

[ShWe] Shannon, C.E., Weaver, W.: The mathematical theory of communication. Urbana, IL: University of Illinois Press 1949

[Shi74] Shimizu, R.: On Fisher's amount of information for location family. In: A modern course on statistical distributions in scientific work. Patil et al. (eds.). Boston, MA: D. Reidel 1974

[Shi82] Shimizu, R.: On the stability of characterizations of the normal distribution. In: Statistics and Probability: Essays in Honor of C.R. Rao. Kallianpur et al. (eds.) pp. 661-670. Amsterdam: North-Holland 1982

[SiSo1] Sigal, I.M., Soffer, A.: Long range many body scattering: Asymptotic clustering for Coulomb type potentials. Inv. Math. 99, 115-143 (1990)

[SiSo2] Sigal, I.M., Soffer, A.: The $N$-particle scattering problem: Asymptotic completeness for short range systems. Ann. Math. 126, 35-108 (1987)

[Sim] Simon, B.: Functional integration and quantum physics. New York: Academic Press 1979

[Ski] Skitovich, V.P.: Linear forms of independent random variables and the normal distribution. Isvestiya Akad. Nauk U.S.S.R. 18, 185-200 (1954)

[Sta] Some inequalities satisfied by the quantities of information of Fisher and Shannon. Info. Contr. 2, 101-112 (1959) 
